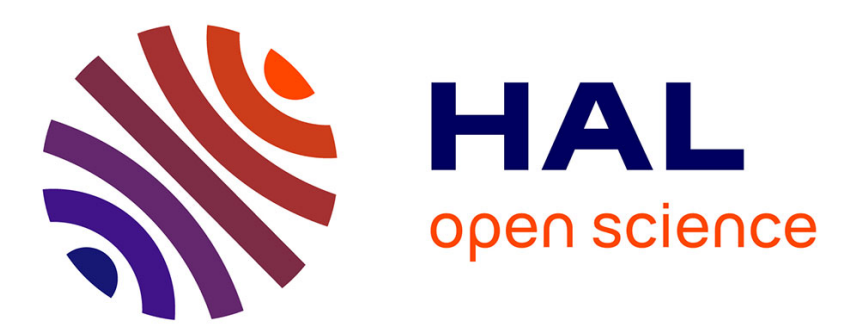

\title{
Stochastic modeling of macromolecules in solution. II. Spectral densities
}

\author{
Antonino Polimeno, Mirco Zerbetto, Daniel Abergel
}

\section{To cite this version:}

Antonino Polimeno, Mirco Zerbetto, Daniel Abergel. Stochastic modeling of macromolecules in solution. II. Spectral densities. Journal of Chemical Physics, 2019. hal-02414649

\author{
HAL Id: hal-02414649 \\ https://hal.science/hal-02414649
}

Submitted on 16 Dec 2019

HAL is a multi-disciplinary open access archive for the deposit and dissemination of scientific research documents, whether they are published or not. The documents may come from teaching and research institutions in France or abroad, or from public or private research centers.
L'archive ouverte pluridisciplinaire HAL, est destinée au dépôt et à la diffusion de documents scientifiques de niveau recherche, publiés ou non, émanant des établissements d'enseignement et de recherche français ou étrangers, des laboratoires publics ou privés. 


\section{Stochastic modeling of macromolecules in solution. II. Spectral densities}

Antonino Polimeno, ${ }^{1}$ a) Mirco Zerbetto, ${ }^{1}$ and Daniel Abergel ${ }^{2}$

1) Dipartimento di Scienze Chimiche, Università degli Studi di Padova, I-35131, Padova, Italy

${ }^{2)}$ Laboratoire des biomolécules, LBM, Département de chimie, Ecole normale supérieure, PSL University, Sorbonne Université, CNRS, 75005 Paris, France

(Dated: 16 December 2019)

In a companion paper ${ }^{1}$ we have proposed a general approach for interpreting relaxation properties of a macromolecule in solution, derived from an atomistic description. A simple model (the semi-flexible Brownian, SFB, approach) has been defined, for the case of limited internal flexibility, but retaining full coupling with external degrees of freedom, inclusion of all of the momenta, and dissipation. Here we discuss the application of the SFB model to the practical evaluation of orientation spectral densities, based on two complementary computational treatments.

a) Electronic mail: antonino.polimeno@unipd.it 


\section{INTRODUCTION}

We discuss in this paper the numerical solution of the semi-flexible Brownian (SFB) model, introduced in the previous paper ${ }^{1}$ (henceforth referred to as I). The model is the simplest outcome of the general derivation presented in I, since it is limited to the case of a molecular system of reduced flexibility assuming the absence of internal motions of large amplitude. This choice implies neglecting activated torsional kinetics and/or crankshaft motions, ${ }^{2,3}$ as well as second order precession effects. Internal motions are described as a harmonic or boson bath. The model retains full coupling with external degrees of freedom and momenta, and includes dissipative/stochastic effects. The SFB model has been derived and discussed in I as an example of description of molecular relaxation processes based on an ab-initio derivation, and takes the form of a multidimensional Fokker-Planck (FP) ${ }^{4}$ equation. Although some of the approximations introduced in I are drastic, the resulting formal appearance of the model remains rather complex. We believe it is relevant to show that computationally efficient methods of solution are available and relatively easy to apply even to systems of large dimensions. The model can then be considered as an effective tool for the interpretation of relaxation processes in several spectroscopic techniques in solution. ${ }^{5-13}$ Moreover, we think it is useful to establish consistent and clear computational methods for this relatively simple model in order to build effective approaches to deal with more advanced descriptions, recoverable within the theoretical framework discussed in I, by lifting some of the approximations introduced to get the SFB model.

The SFB approach is at least comparable to most phenomenological methods of interpretation available in the literature (see I and references quoted therein), and can be used as a first coarse-grained description. In practice, one can use some of the semi-analytical expressions and results obtained in this work as tools to gather quick information of the dynamical behavior of molecular systems. Also, in this work we validate our computational approach, and the obtained results and conclusions compare well with data obtained directly from molecular dynamics simulations. Finally, this approach stands per se and can be used as a purely interpretative tool. We further discuss this point in the last section of the paper.

This work is organized as follows. In Section II we summarize the basic features of the model, and present the general form of the FP operator. We then discuss in Section III an analytical approximate solution for the case of fast internal relaxation. In Section IV a 
semi-quantitative solution is presented based on a moment expansion, which allows for the quick evaluation of correlation function and spectral densities. ${ }^{14,15}$ Results are summarized and discussed in Section V.

\section{THE MODEL}

The SFB model ${ }^{1}$ is based on the FP equation for the probability density $\rho(\mathcal{Q}, t)$ (eq. 52 of I):

$$
\begin{aligned}
\frac{\partial \rho(\mathcal{Q}, t)}{\partial t} & =-\hat{\Gamma} \rho(\mathcal{Q}, t) \\
\hat{\Gamma} & =\hat{\Gamma}_{0}+\hat{\Gamma}_{\text {int }}=-\sum_{i, j=1}^{N} \omega_{i j}^{\text {io }} \frac{\partial}{\partial x_{i}} p(\mathbf{x}) \frac{\partial}{\partial x_{j}} p(\mathbf{x})^{-1}+\sum_{i=1}^{N} \sum_{p=1}^{3} \omega_{i p}^{\text {int }} x_{i} \hat{M}_{p}
\end{aligned}
$$

In this equation, $\mathcal{Q}=(\Omega, \mathbf{x})$, where $\Omega$ denotes the set of Euler angles describing the orientation of a molecular frame (MF, see I for details) with respect to a fixed frame, $\mathbf{x}=\left(x_{1}, \ldots, x_{i}, \ldots, x_{N}\right)$ is a set of dimensionless harmonic degrees of freedom, obtained as linear combinations of internal coordinates, their conjugate momenta, and external (angular) momentum components. $\hat{M}_{p}, p=1,2,3$, are the components of the infinitesimal rotation operator in the MF. ${ }^{16}$ The model describes the semi-rigid macromolecule of $n$ atoms (or extended atoms, when a coarse grained representation of the molecule is used) as a rotator coupled to $N=6 n-9$ (i.e. $3 n-6$ internal coordinates, $3 n-6$ internal momenta and 3 components of the angular momentum $\mathbf{L}$ vector) harmonic degrees of freedom, in a fashion quite similar to standard spin-boson quantum mechanical approaches. Notice however the additional complexity with respect to quantum spin-boson systems, ${ }^{17-22}$ since we deal with non-Hermitian operators. Here $p(\mathbf{x})=\exp \left(-x^{2} / 2\right) /(2 \pi)^{N / 2}$ is the equilibrium distribution of the $N$ modes (a multidimensional Gaussian function) and the equilibrium distribution is $\rho(\mathcal{Q})=p(\mathbf{x}) / 8 \pi^{2} ; \omega_{i j}^{\text {io }}$ is a (non-symmetric) matrix describing the relaxation of the internal coordinates, while $\omega_{i p}^{\text {int }}$ dictates the coupling between rotation and internal coordinates. Both $\omega_{i j}^{\text {io }}$ and $\omega_{i p}^{\text {int }}$ can be derived from the molecular geometry. ${ }^{1}$

We want to evaluate the spectral densities associated to orientation correlation functions $G(t)=\langle f(\Omega)|\exp (-\hat{\Gamma} t)| g(\Omega) \rho(\mathcal{Q})\rangle_{\mathcal{Q}}$ relative to a given molecular frame (cfr. I) and defined by a set of Euler angles $\Omega .{ }^{16}$ Here and in the following, integration with respect to $\mathbf{x}, \Omega$ and both sets of coordinates will be respectively denoted with $\langle\ldots\rangle=\int d \mathbf{x},\langle\ldots\rangle_{\Omega}=\int d \Omega$ and 
$\langle\ldots\rangle_{\mathcal{Q}}=\left\langle\langle\ldots\rangle_{\Omega}\right\rangle$. The most common choice for the functions $f$ and $g$, in the case of nuclear magnetic resonance experiments, is given by second rank Wigner matrix functions, $\mathcal{D}_{0, l}^{2}(\Omega)$, weighted by suitable normalization coefficients. ${ }^{1}$ In practice we shall focus on correlation functions and spectral densities defined as:

$$
\begin{aligned}
& G_{l \bar{l}}(t)=5\left\langle\mathcal{D}_{0, l}^{2}(\Omega)^{*}|\exp (-\hat{\Gamma} t)| \mathcal{D}_{0, \bar{l}}^{2}(\Omega) \rho\right\rangle_{\mathcal{Q}} \\
& J_{l \bar{l}}(\omega)=\int_{0}^{\infty} d t \exp (-i \omega t) G_{l \bar{l}}(t)=5\left\langle\mathcal{D}_{0, l}^{2}(\Omega)^{*}\left|(i \omega+\hat{\Gamma})^{-1}\right| \mathcal{D}_{0, \bar{l}}^{2}(\Omega) \rho\right\rangle_{\mathcal{Q}}
\end{aligned}
$$

with $l, \bar{l}=0, \pm 1, \pm 2$, which arise naturally in nuclear magnetic resonance relaxation experiments. ${ }^{1,5-7}$ For the sake of convenience in further derivations, we shall evaluate the $J_{l, l}(\omega)$ as linear combinations of spectral densities of elements of the orthonormal set of real functions:

$$
S_{l}(\Omega)=\sqrt{\frac{5}{8 \pi^{2}}} \times\left\{\begin{array}{cc}
\frac{1}{\sqrt{2}}\left[\mathcal{D}_{0, l}^{2}(\Omega)+(-1)^{l} \mathcal{D}_{0,-l}^{2}(\Omega)\right] & 0<l \leq 2 \\
\mathcal{D}_{0,0}^{2}(\Omega) & l=0 \\
\frac{i}{\sqrt{2}}\left[\mathcal{D}_{0, l}^{2}(\Omega)-(-1)^{l} \mathcal{D}_{0,-l}^{2}(\Omega)\right] & -2 \leq l<0
\end{array}\right.
$$

Due to the form of the time evolution operator $\hat{\Gamma}$ these functions (or their original complex counterparts $\mathcal{D}_{0, l}^{2}(\Omega)$ ) form a closed set (since $\hat{M}_{p}$ couples only the index $l$ ). Notice that for generic observables of rank $L$, the previous expressions can be generalized without difficulty to a $2 L+1$ subspace. In practice we define the normalized correlation functions:

$$
g_{\bar{l}}(t)=8 \pi^{2}\left\langle S_{l}|\exp (-\hat{\Gamma} t)| S_{\bar{l}} \rho\right\rangle_{\mathcal{Q}}
$$

where $g_{l \bar{l}}(0)=\delta_{l \bar{l}}$, with $l, \bar{l}=-2, \ldots, 2$; the related spectral densities are:

$$
j_{l \bar{l}}(\omega)=8 \pi^{2}\left\langle S_{l}\left|(i \omega+\hat{\Gamma})^{-1}\right| S_{\bar{l}} \rho\right\rangle_{\mathcal{Q}}
$$

The $J_{l l}$ 's are obtained as linear combinations of the $j_{l l}$ 's, listed in Appendix D. 


\section{ASYMPTOTIC REGIME: PROJECTION OF INTERNAL COORDINATES}

In the limit of fast relaxing internal coordinates, we can resort conveniently to projection techniques. A detailed review of the Nakajima-Zwanzig ${ }^{14,15}$ approach for the projection of fast coordinates in the time evolution Fokker-Planck operators is given in I. Here we specialize the procedure in the frequency domain, and propose an advanced perturbation scheme for the direct evaluation of spectral densities. Let us first consider the derivation of a general equation for the reduced density:

$$
\rho(\Omega, t)=\langle\rho(\mathcal{Q}, t)\rangle
$$

We define the projection operator $\hat{P} \ldots=p(\mathbf{x})\langle\ldots\rangle$. In line with other similar treatments, ${ }^{14,15}$ we assume that $\Omega$ and $\mathbf{x}$ are initially statistically uncorrelated, so that the initial phase density is $\rho(\mathcal{Q}, 0)=\rho(\Omega, 0) p(\mathbf{x})$. This is also in agreement with the requirements for the calculation of correlation functions of the type shown in Eq. 6. Defining $\hat{Q}=1-\hat{P}$, the well-known formal solution is obtained (see I):

$$
\begin{aligned}
\frac{\partial \hat{P} \rho(\mathcal{Q}, t)}{\partial t} & =-\hat{P} \hat{\Gamma} \hat{P} \rho(\mathcal{Q}, t) \\
& +\int_{0}^{t} d \tau \hat{P} \hat{\Gamma} \exp (-\hat{Q} \hat{\Gamma} \tau) \hat{Q} \hat{\Gamma} \hat{P} \rho(\mathcal{Q}, t-\tau)+\hat{P} \hat{\Gamma} \exp (-\hat{Q} \hat{\Gamma} t) \hat{Q} \rho(\mathcal{Q}, 0)
\end{aligned}
$$

The first term is the zero-order averaged operator, the second term is defined as a functional of a general kernel operator that is a function of time, and the third term depends on the initial conditions. Since $\hat{P} \rho(\mathcal{Q}, 0)=\rho(\mathcal{Q}, 0)$ the last term goes to zero. Rearranging, ${ }^{1}$ one finds:

$$
\frac{\partial \rho(\Omega, t)}{\partial t}=-\left\langle\hat{\Gamma}_{0} p(\mathbf{x})\right\rangle \rho(\Omega, t)+\int_{0}^{t}\left\langle\hat{\Gamma}_{\mathrm{int}} \exp (-\hat{Q} \hat{\Gamma} \tau) \hat{Q} \hat{\Gamma}_{\mathrm{int}} p(\mathbf{x})\right\rangle \rho(\Omega, t-\tau) d \tau
$$

where the equation $\hat{P} \hat{\Gamma}=\hat{\Gamma}_{\text {int }}$ is used. Although it is not essential, it is useful to introduce symmetrized forms of the original and projected operators. We define the symmetrized probability $\tilde{\rho}(\mathcal{Q}, t)=\rho(\mathcal{Q}, t) \rho(\mathcal{Q})^{-1 / 2}$ and operators $\tilde{\Gamma}=\rho(\mathcal{Q})^{-1 / 2} \hat{\Gamma} \rho(\mathcal{Q})^{1 / 2}=\tilde{\Gamma}_{0}+\tilde{\Gamma}_{\text {int }}$,

$\tilde{\Gamma}_{0}=p(\mathbf{x})^{-1 / 2} \hat{\Gamma}_{0} p(\mathbf{x})^{1 / 2}, \tilde{\Gamma}_{\text {int }}=\hat{\Gamma}_{\text {int }}$. We can introduce a convenient Dirac notation $|\mathbf{0}\rangle=$ $p(\mathbf{x})^{1 / 2}$ and $\tilde{\Gamma}_{0}|\mathbf{0}\rangle=0$, i.e. $|\mathbf{0}\rangle$ is the eigenvector with null eigenvalue of $\tilde{\Gamma}_{0}$. In fact $\tilde{\Gamma}_{0}$ and 
its adjoint $\tilde{\Gamma}_{0}^{\dagger}$ define a bi-orthonormal set of states

$$
\begin{aligned}
\tilde{\Gamma}_{0}|\mathbf{n}\rangle & =\Lambda_{\mathbf{n}}|\mathbf{n}\rangle \\
\tilde{\Gamma}_{0}^{\dagger}|\overline{\mathbf{n}}\rangle & =\Lambda_{\mathbf{n}}^{*}|\overline{\mathbf{n}}\rangle \\
\left\langle\overline{\mathbf{n}} \mid \mathbf{n}^{\prime}\right\rangle & =\delta_{\mathbf{n}, \mathbf{n}^{\prime}}
\end{aligned}
$$

with $|\mathbf{0}\rangle=|\overline{\mathbf{0}}\rangle, \Re \Lambda_{\mathbf{n}}>0$ for $\mathbf{n} \neq \mathbf{0}$. In Appendix A the general properties of $\tilde{\Gamma}_{0}$ are summarized. The symmetrized projection operator takes the form $\tilde{P}=|\mathbf{0}\rangle\langle\overline{\mathbf{0}}|$ and $\tilde{Q}=1-\tilde{P}$. The reduced symmetrized density is $\tilde{\rho}(\Omega, t)=\rho(\Omega, t)\left(8 \pi^{2}\right)^{1 / 2}$, and the reduced equilibrium density is $\tilde{\rho}(\Omega)=\left(8 \pi^{2}\right)^{-1 / 2}$. The symmetrized counterpart of Eqs. 9 for the time evolution of $\tilde{\rho}(\Omega, t)$ is:

$$
\frac{\partial \tilde{\rho}(\Omega, t)}{\partial t}=-\left\langle\overline{\mathbf{0}}\left|\tilde{\Gamma}_{0}\right| \mathbf{0}\right\rangle \tilde{\rho}(\Omega, t)+\int_{0}^{t}\left\langle\overline{\mathbf{0}}\left|\tilde{\Gamma}_{\text {int }} \exp (-\tilde{Q} \tilde{\Gamma} \tau) \tilde{Q} \tilde{\Gamma}_{\text {int }}\right| \mathbf{0}\right\rangle \tilde{\rho}(\Omega, t-\tau) d \tau
$$

The symmetrized form of a generic correlation function is:

$$
G(t)=\left\langle f(\Omega) \rho^{1 / 2}(\mathcal{Q})|\exp (-\tilde{\Gamma} t)| g(\Omega) \rho^{1 / 2}(\mathcal{Q})\right\rangle_{\mathcal{Q}}=\left\langle f(\Omega) \rho^{1 / 2}(\mathcal{Q}) \mid \tilde{\rho}(\mathcal{Q}, t)\right\rangle_{\mathcal{Q}}
$$

where $\tilde{\rho}(\mathcal{Q}, t)$ is the symmetrized density evolved from the initial condition $\rho(\Omega, 0)=$ $g(\Omega) \tilde{\rho}(\Omega)$. Using 11 and taking a Fourier-Laplace transform, we can obtain the spectral density associated to $G(t)$ directly as

$$
J(\omega)=\left\langle f(\Omega)\left|[i \omega+\hat{G}(\omega)]^{-1}\right| g(\Omega)\right\rangle_{\Omega} / 8 \pi^{2}
$$

where the external average is taken on the orientation variables only, and the reduced evolution operator is:

$$
\hat{G}(\omega)=\left\langle\overline{\mathbf{0}}\left|\tilde{\Gamma}_{\text {int }}\right| \mathbf{0}\right\rangle-\left\langle\overline{\mathbf{0}}\left|\tilde{\Gamma}_{\text {int }}(i \omega+\tilde{Q} \tilde{\Gamma})^{-1} \tilde{Q} \tilde{\Gamma}_{\text {int }}\right| \mathbf{0}\right\rangle
$$

Eq. 13 is exact, but deceptively simple. As it is often the case with projection procedures, additional effort is required to make it usable in practical applications. Following several authors ${ }^{17-22}$ we propose here a possible route, based on a well established procedure for quantum Liouville equations, ${ }^{23}$ which is here extended to non-Hermitian multidimensional 
FP operators. We start with the Dyson identity, valid for any two generic operators $\hat{A}, \hat{B}$ :

$$
(\hat{A}+\hat{B})^{-1}=\hat{A}^{-1}\left[1-\hat{B}(\hat{A}+\hat{B})^{-1}\right]
$$

which can be easily proved by inspection. We apply now the identity to $(i \omega+\tilde{Q} \tilde{\Gamma})^{-1}$

$$
\begin{aligned}
(i \omega+\tilde{Q} \tilde{\Gamma})^{-1} & =\left(i \omega+\tilde{\Gamma}_{0}+\tilde{Q} \tilde{\Gamma}_{\text {int }}\right)^{-1} \\
& =\left(i \omega+\tilde{\Gamma}_{0}\right)^{-1}\left[1-\tilde{Q} \tilde{\Gamma}_{\text {int }}\left(i \omega+\tilde{\Gamma}_{0}+\tilde{Q} \tilde{\Gamma}_{\text {int }}\right)^{-1}\right]
\end{aligned}
$$

where the identity $\tilde{P} \tilde{\Gamma}_{0}=0$ has been used. The previous expression can be used to recast $(i \omega+\tilde{Q} \tilde{\Gamma})^{-1}$ in a more convenient form

$$
\begin{aligned}
(i \omega+\tilde{Q} \tilde{\Gamma})^{-1} & =\left[1+\left(i \omega+\tilde{\Gamma}_{0}\right)^{-1} \tilde{Q} \tilde{\Gamma}_{\mathrm{int}}\right]^{-1}\left(i \omega+\tilde{\Gamma}_{0}\right)^{-1} \\
& =\sum_{k=0}^{\infty}(-1)^{k}\left[\left(i \omega+\tilde{\Gamma}_{0}\right)^{-1} \tilde{Q} \tilde{\Gamma}_{\mathrm{int}}\right]^{k}\left(i \omega+\tilde{\Gamma}_{0}\right)^{-1}
\end{aligned}
$$

Substituting in Eq. 13 and rearranging terms one gets

$$
\hat{G}(\omega)=\sum_{k=0}^{\infty}(-1)^{k}\left\langle\overline{\mathbf{0}}\left|\tilde{\Gamma}_{\mathrm{int}}\left[\left(i \omega+\tilde{\Gamma}_{0}\right)^{-1} \tilde{Q} \tilde{\Gamma}_{\mathrm{int}}\right]^{k}\right| \mathbf{0}\right\rangle_{I}=\sum_{k=0}^{\infty} \hat{g}_{k}(\omega)
$$

this is essentially a perturbation expansion where the $k$-th term $\hat{G}_{k}(\omega)$ is of order $k+1$ in $\tilde{\Gamma}_{\text {int }}$. Formally, this expression is equivalent to a total time ordered cumulant (TTOC) expansion. ${ }^{24}$

It is possible (see Appendix B) to write Eq. 18 as an effective sum over operators acting only on the orientation variables. The main result is that for $l \geq 0, \hat{g}_{2 l}=0$ and

$$
\hat{g}_{2 l+1}(\omega)=-\sum_{p_{1}, \ldots, p_{2(l+1)}}{ }^{[2(l+1)]} D_{p_{1} \ldots p_{2(l+1)}}(\omega) \hat{M}_{p_{1}} \ldots \hat{M}_{p_{2(l+1)}}
$$

where $\hat{M}_{p_{i}}$ is the $p_{i}$-th Cartesian component in the MF of the vector operator $\hat{\mathbf{M}}$ and the elements ${ }^{[2 l]} D_{p_{1} \ldots p_{2 l}}(\omega)$ of the generalized $\omega$-dependent 'diffusion' Cartesian tensor of rank $2 l$ are obtained as explicit functions of parameters $\omega_{i j}^{\text {io }}, \omega_{i p}^{\text {int }}$. Therefore $\hat{G}(\omega)$ is obtained in the 
form

$$
\hat{G}(\omega)=-\sum_{p_{1} p_{2}}^{[2]} D_{p_{1} p_{2}}(\omega) \hat{M}_{p_{1}} \hat{M}_{p_{2}}-\sum_{p_{1} p_{2} p_{3} p_{4}}{ }^{[4]} D_{p_{1} p_{2} p_{3} p_{4}}(\omega) \hat{M}_{p_{1}} \hat{M}_{p_{2}} \hat{M}_{p_{3}} \hat{M}_{p_{4}}-\ldots
$$

In practice, one can show (cfr. Appendix B) that the second order tensor ${ }^{[2]} D_{p_{1} p_{2}}(\omega)$ can be obtained directly from $\omega_{i j}^{\text {io }}, \omega_{i p}^{\text {int }}$, which writes, in matrix form:

$$
{ }^{[2]} \mathbf{D}(\omega)=\left(\boldsymbol{\omega}^{\text {int }}\right)^{\operatorname{tr}}\left(i \omega \mathbf{1}+\boldsymbol{\omega}^{\text {io }}\right)^{-1} \boldsymbol{\omega}^{\text {int }}
$$

For $\omega \rightarrow 0,{ }^{[2]} \mathbf{D}(\omega)$ tends to the real tensor $\left(\boldsymbol{\omega}^{\mathrm{int}}\right)^{\mathrm{tr}} \boldsymbol{\omega}^{\mathrm{io}}{ }^{-1} \boldsymbol{\omega}^{\mathrm{int}}$.

From Eq. 20 one can obtain a numerical estimate of Eq. 6 in matrix form:

$$
\mathbf{J}(\omega)=[i \omega \mathbf{1}+\mathbf{G}(\omega)]^{-1}
$$

where $J_{l \bar{l}}(\omega)$ is the $(l, \bar{l})$ element of the $5 \times 5$ matrix $[i \omega \mathbf{1}+\mathbf{G}(\omega)]^{-1}$. In the following we discuss results obtained truncating the expansion of $\hat{G}$ at $k=1(l=0)$ and $k=3(l=1)$, since the evaluation of higher terms is complicate. In the case $k=3$, Eq. 6 can be written in matrix form as:

$$
\mathbf{G}(\omega)=-\sum_{p_{1} p_{2}}^{[2]} D_{p_{1} p_{2}}(\omega) \mathbf{M}_{p_{1}} \mathbf{M}_{p_{2}}-\sum_{p_{1} p_{2} p_{3} p_{4}}{ }^{[4]} D_{p_{1} p_{2} p_{3} p_{4}}(\omega) \mathbf{M}_{p_{1}} \mathbf{M}_{p_{2}} \mathbf{M}_{p_{3}} \mathbf{M}_{p_{4}}
$$

where the $(l, \bar{l})$ matrix element of the rotational operator $\mathbf{M}_{p}$ are $M_{p, l \bar{l}}=\left\langle S_{l}\left|\hat{M}_{p}\right| S_{\bar{l}}\right\rangle_{\Omega}$, and are found immediately from well-known properties of the Wigner matrix functions (see Appendix D). ${ }^{16}$

\section{MOMENTS}

The approximate expression 22 obtained in the previous Section is valid in a limited dynamical range, from fast $(k=1)$ to moderately fast $(k=3)$ relaxation of $\mathbf{x}$. More general solutions would require the evaluation of higher orders terms of Eq. 19, but the ex-

pressions, although manageable using symbolic algebra software, are quite cumbersome. An exact evaluation of $\mathbf{J}(\omega)$ via direct numerical solution via traditional approaches (variational 
methods, finite elements or difference approaches) is already quite demanding for relatively small molecules: already in the case of the smaller system treated in I, an alanine dimer, the number of internal modes $N$ is equal to 209 (see Section V). As an alternative, we propose a quantitative treatment based on a moment expansion. Although still an approximation, this approach has several merits: it does not depend upon the hypothesis of different orientation and internal time-scales and it allows a computationally cheap evaluation of correlation functions and spectral densities without resorting to a complex perturbation expansion. To proceed, we start from the non-symmetrized definition of the correlation function, Eq. 5. To evaluate it we need to solve the original time evolution Eq. 1 for $\rho(\mathcal{Q}, t)$ with initial condition $\rho(\mathcal{Q}, 0)=8 \pi^{2} S_{\bar{l}}(\Omega) \rho=S_{\bar{l}}(\Omega) p(\mathbf{x})$; we can write

$$
\rho(\mathcal{Q}, t)=\sum_{l} S_{l}(\Omega) \rho_{l}(\mathbf{x}, t)
$$

with initial conditions

$$
\rho_{l}(\mathbf{x}, 0)=\delta_{l \bar{l}}
$$

The time evolution equation for the $2 L+1$ unknown functions $\rho_{l}$ are obtained simply multiplying for a generic $S_{l}$ and integrating upon $\Omega$

$$
\frac{\partial}{\partial t} \rho_{l}(\mathbf{x}, t)=\sum_{i j} \omega_{i j}^{\text {io }} \frac{\partial}{\partial x_{i}} p(\mathbf{x}) \frac{\partial}{\partial x_{j}} p(\mathbf{x})^{-1} \rho_{l}(\mathbf{x}, t)-\sum_{i, \bar{l}} \bar{\omega}_{i, l \bar{l}}^{\text {int }} x_{i} \rho_{\bar{l}}(\mathbf{x}, t)
$$

where $\bar{\omega}_{i, l \bar{l}}^{\text {int }}=\sum_{p}{ }^{S} M_{p, l l} \omega_{i p}^{\text {int }}$. We find now closed expressions for the zeroth, first, second moments of functions $\rho_{l}(\mathbf{x}, t)$

$$
\begin{aligned}
& M_{l}^{(0)}=\left\langle\rho_{l}(\mathbf{x}, t)\right\rangle \\
& \mathbf{M}_{l}^{(1)}=\left\langle\mathbf{x} \rho_{l}(\mathbf{x}, t)\right\rangle \\
& \mathbf{M}_{l}^{(2)}=\left\langle\mathbf{x x}^{\operatorname{tr}} \rho_{l}(\mathbf{x}, t)\right\rangle
\end{aligned}
$$

i.e. the averages with respect to internal variables of functions $\rho_{l}(\mathbf{x}, t), x_{m} \rho_{l}(\mathbf{x}, t), x_{m} x_{n} \rho_{l}(\mathbf{x}, t)$. A system of first order linear differential equations for the time evolution of the moments is 
found by direct evaluation of their time derivatives. One gets

$$
\begin{aligned}
& \dot{M}_{l}^{(0)}=-\sum_{\bar{l}} \boldsymbol{\omega}_{l \bar{l}}^{\text {int }} \mathbf{M}_{\bar{l}}^{(1)} \\
& \dot{\mathbf{M}}_{l}^{(1)}=-\boldsymbol{\omega}^{\text {io }} \mathbf{M}_{l}^{(1)}-\sum_{\bar{l}} \mathbf{M}_{\bar{l}}^{(2)} \boldsymbol{\omega}_{l \bar{l}}^{\text {int }} \\
& \dot{\mathbf{M}}_{l}^{(2)}=\left(\boldsymbol{\omega}^{\mathrm{io}}+\boldsymbol{\omega}^{\mathrm{iot}}\right) M_{l}^{(0)}-\left(\boldsymbol{\omega}^{\text {io }} \mathbf{M}_{l}^{(2)}+\mathbf{M}_{l}^{(2)} \boldsymbol{\omega}^{\text {io }}{ }^{\mathrm{tr}}\right)+\text { higher order moments }
\end{aligned}
$$

with $\dot{f}=d f / d t$. We choose to close approximately the system by neglecting $3^{r d}$ and higher order moments. We are left with a set of $5\left(1+N+N^{2}\right)$ linear differential equations actually $5[1+N+N(N+1) / 2]$ taking into account the symmetric nature of $\mathbf{M}_{l}^{(2)}(t)$. Initial conditions, based on Eq. 25, are

$$
\begin{aligned}
& M_{l}^{(0)}(0)=\delta_{l \bar{l}} \\
& \mathbf{M}_{l}^{(1)}(0)=\mathbf{0} \\
& \mathbf{M}_{l}^{(2)}(0)=\mathbf{1} \delta_{l \bar{l}}
\end{aligned}
$$

Notice that in terms of moments the correlation functions defined in Eq. 5 are simply $G_{\bar{l}}(t)=$ $M_{l}^{(0)}$ and likewise, spectral densities defined in Eq. 6 are found as Fourier-Laplace transforms of the zero-th order moments. The solution of Eqs. 29 can be found straightforwardly by vectorizing the unknown $\mathbf{X}=\left(M_{-2}^{(0)}, \ldots, M_{2}^{(0)}, M_{1,-2}^{(1)}, \ldots, M_{N, 2}^{(1)}, M_{1,1,-2}^{(2)}, \ldots, M_{N, N, 2}^{(2)}\right)$ and recasting Eqs. 29 as

$$
\dot{\mathbf{X}}=\mathbf{A X}
$$

where $\mathbf{A}$ is a constant $5\left(1+N+N^{2}\right) \times 5\left(1+N+N^{2}\right)$ block-sparse matrix (see below and Appendix D). Alternatively, and much more conveniently, if one is interested directly in the spectral densities, we can deflate the rather large dimension of the problem. In Appendix $\mathrm{C}$ we give the details of the derivation. The final result is

$$
\mathbf{J}(\omega)=[i \omega \mathbf{1}-\boldsymbol{\beta}(\omega)]^{-1}[\mathbf{1}+\boldsymbol{\gamma}(\omega)]
$$


where the elements of $5 \times 5$ matrices $\boldsymbol{\beta}, \boldsymbol{\gamma}$ are

$$
\begin{aligned}
& \beta_{l l^{\prime}}=\sum_{l^{\prime \prime} i j} e_{i, l l^{\prime \prime}} \frac{S_{i j}\left(\lambda_{i}+\lambda_{j}\right)}{\left(i \omega+\lambda_{i}\right)\left(i \omega+\lambda_{i}+\lambda_{j}\right)} e_{j, l^{\prime \prime} l^{\prime}} \\
& \gamma_{l l^{\prime}}=\sum_{l^{\prime \prime} i j} e_{i, l l^{\prime \prime}} \frac{S_{i j}}{\left(i \omega+\lambda_{i}\right)\left(i \omega+\lambda_{i}+\lambda_{j}\right)} e_{j, l^{\prime \prime} l^{\prime}}
\end{aligned}
$$

where $\lambda_{i}, S_{i j}, e_{i, l l^{\prime}}$ are obtained from $\boldsymbol{\omega}^{\text {io }}, \boldsymbol{\omega}^{\text {int }}$ (cfr. Appendices A, C).

\section{RESULTS AND DISCUSSION}

We present some selected results on two model molecular systems, already considered in I, namely the poly-alanines $\mathbf{2}$ (2-alanine) and $\mathbf{8}$ (8-alanine), cfr. Fig. 1. The two molecular structures are chosen to respectively illustrate the cases of a small, relatively rigid molecule (2) and a more flexible, larger one (8). A complete validation of our approach as an alternative to other existing methods for describing and rationalizing relaxation processes in macromolecules (see the Introduction section in I) requires extensive comparisons and analyses, which is beyond the goal of the present study, and will be the subject of further work, where the extension of the SFB approach will also be discussed. Here, we focus on methods

of solutions that are not conventional and that will serve as the basis for further explorations, and we discuss the theoretical framework described in I. In particular, a discussion concerning the validation of the approach is provided at the end of this Section.

\section{A. Parametrization}

Parametrization of a stochastic model like SFB can be set up at different levels. In I we compared the internal coordinates free energy surface obtained via covariance-variance matrix computed from short MD simulations versus the one obtained directly from a single Hessian calculated with respect to a reference structure. We also defined a hydrodynamic approach to estimate the friction tensor. Other, more computationally costly, choices, are possible, and likely necessary, in order to achieve quantitative comparison with experimental relaxation data performed on large flexible macromolecules. For instance, short biased MD simulations may become necessary to parameterize the free energy of the system when large amplitude and/or activated processes are included. Here, we consider the case of $\mathbf{2}$ 
and 8 molecules, mainly fluctuating around a global energy minimum. Therefore, we shall dispense completely from MD-derived parameters, and instead make use of the Hessian matrix computed around the global energy minimum to estimate the free energy. ${ }^{1}$ Thus, the following parameterization protocol have been employed: $i$ ) the molecular system has been energy minimized in vacuo using the Amber99 force field ${ }^{25}$ and the MMTK software, ${ }^{26}$ ii) the Hessian of the energy has been computed in internal coordinates; ${ }^{27}$ iii) the friction matrix has been obtained via a simplified hydrodynamic approach; $\left.{ }^{1} i v\right) \boldsymbol{\omega}^{\text {io }}$ and $\boldsymbol{\omega}^{\text {int }}$ have been calculated following Section IV.A of Paper I. Further technical details are summarized in Appendix E.

\section{B. Evaluation}

Numerical evaluation of the spectral densities, even for the larger system $(N=489)$ requires roughly half an hour on a serial desktop CPU via the moments methods, and a hour via the perturbation method (at $k=3$ order); optimization of the $\mathrm{C}++$ code employed (parallelization of multiple sums in the perturbation method mainly) and additional approximations (e.g. cutting off some of the internal modes) could reduce the time of at least one order of magnitude.

In Fig. 2, the spectral density $J_{00}(\omega)$ calculated by both the perturbation and the moment methods are shown. The real (positive curves) and imaginary (negative curves) parts of $J_{00}(\omega)$ are depicted in (a) and (b) for $\mathbf{2}$ and $\mathbf{8}$, respectively. Results from both perturbation orders are shown. The lower order asymptotic expression of Eq. 22 (dotted lines) describes, in essence, the spectral density resulting from a rigid body with a 'dressed' diffusion tensor as in Eq. 21, while the fourth-order expression (dashed lines) is based on Eq. 23. The moment-based expression of Eq. 31 (solid lines) confirms that faster components are barely present in the smaller rigid system $\mathbf{2}$, but become relevant for the larger polypeptide $\mathbf{8}$. This fact is illustrated in Fig. 3, where the Cole-Cole plots of $J_{00}(\omega)$ for $\mathbf{2}$ (a) and 8 (b) are shown. The picture emerging from this first analysis is a (relatively) familiar one: the overall tumbling is a slow relaxing process accompanied by faster motions; this causes a decrease of the value of $J_{00}(0)=\tau_{\text {corr }}$ with respect to the value predicted by rigid tumbling alone.

To verify quantitatively this statement, we can look first at the components of the dressed

diffusion tensor at $\omega=0$, i.e. ${ }^{[2]} D_{i j}(0)$, in Table I; notice that ${ }^{[2]} D_{i j}(0)$ is symmetric in 
TABLE I. Dressed diffusion tensor components ${ }^{[2]} D_{i j}(0)$

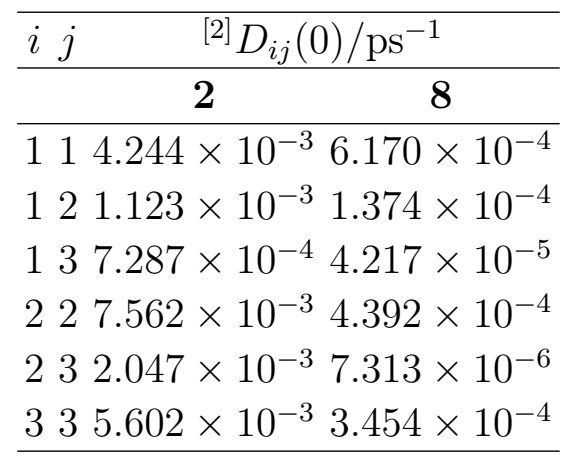

practice, although $\boldsymbol{\omega}$ is not. The principal values of ${ }^{[2]} D_{i j}(0)$ can be obtained, Table II, and they can be compared with the predicted values of the model used in the first place to evaluate the hydrodynamic friction tensor, as a test of the self-consistency of the methods.

TABLE II. Principal values $D_{i}$ obtained after diagonalization of the dressed diffusion tensor ${ }^{[2]} D_{i j}(0)$, and values $D_{i}^{\text {hydro }}$ obtained diagonalizing the diffusion tensor evaluated from the hydrodynamic approach

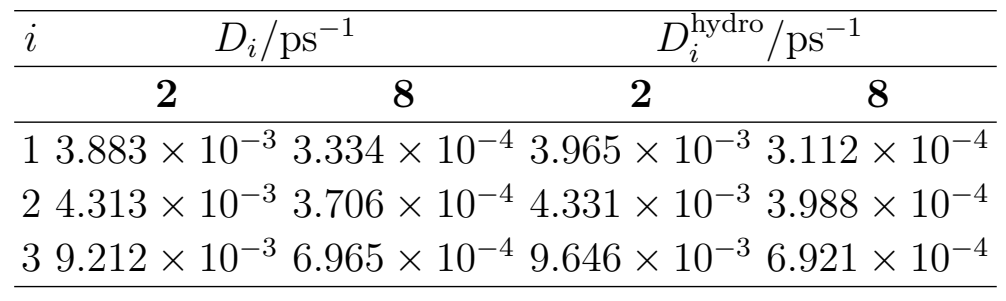

For a rigid rotator characterized by the diffusion tensor ${ }^{[2]} \mathbf{D}(0)$, one can also calculate the exact decay frequencies $d_{i}$ simply by diagonalizing the $5 \times 5$ matrix $\mathbf{G}(0)$ obtained from Eq. 23 setting $\omega=0$. The frequencies are reported in Table III.

TABLE III. Eigenvalues $d_{i}$ of $\mathbf{G}(0)$

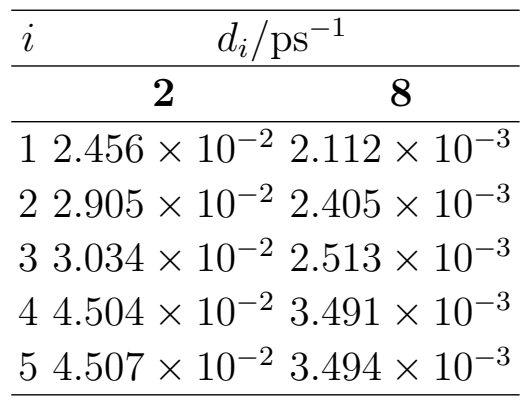

The presence of multi-scale relaxation can be analyzed in more detail by looking at the eigenvalues of matrix $\mathbf{A}$, which constitute the spectrum of decay frequencies of the spectral densities. The spectrum of $\mathbf{A}$ is characterized by the presence of clusters of real eigenvalues, 
increasing in magnitude from values comparable to $d_{i}$ up to much higher values that are directly related to internal fast relaxation modes. Complex eigenvalues are also numerous, due to the libration inertial motions of the internal coordinates (cfr. I). In Fig. 4 the first 50 real eigenvalues are plotted for $\mathbf{2}$ (a) and $\mathbf{8}(\mathrm{b})$; the horizontal lines indicate the corresponding values of $d_{i}$. These observations are confirmed by the analysis of other spectral densities. In Figs. 5 and 6 we show the initial trend of real and imaginary parts of $J_{l \bar{l}}(\omega)$ with $l, \bar{l}=0, \pm 2$ for structure 2 .

TABLE IV. Values of $J_{l, l}(0) /$ ps for the three diagonal spectral densities with $l=0, \pm 2$, obtained using different strategies of solution: the method of moments, Eqs. 28-32), and the perturbation method, Eq. 22, truncated at $k=1$ and $k=3$ order. In parentheses, in the $k=3$ column, the relative error with respect to the solution based on the moments is reported.

\begin{tabular}{cccc}
\hline$l$ & Moments $k=1$ & $k=3$ \\
\hline \multicolumn{4}{c}{ molecule 2} \\
\hline-2 & 28.94 & 30.59 & $27.48(-5 \%)$ \\
0 & 27.60 & 29.59 & $25.79(-7 \%)$ \\
2 & 29.46 & 31.10 & 28.01 \\
\hline \multicolumn{4}{c}{ molecule 8} \\
\hline-2 & 213.1 & 423.6 & $206.0(-3 \%)$ \\
0 & 216.5 & 329.4 & $191.2(-12 \%)$ \\
2 & 211.7 & 416.3 & $205.4(-3 \%)$ \\
\hline
\end{tabular}

\section{Comparison with MD}

We compare here some results of the SFB approach, for the model systems described, with the same quantities obtained directly from all-atom MD simulations. We would like to stress that direct evaluation of orientational correlation functions and, above all, spectral densities for semi-flexible and flexible molecular systems, is not, even with existing powerful computational resources, a trivial task, especially if a good accuracy is requested on both short and long timescales. Spectral densities obtained by a stochastic model like SFB or others account for long times dynamics and fast relaxation processes in an approximated, but complete way. In order to compute the same information from an all-atom MD simulation, one needs to produce long trajectories sampled very frequently. For the case of $\mathbf{2}$ and $\mathbf{8}$, we calculated correlation functions from MD simulations trajectories using the same 
force field and setup as reported in Paper I. The two major differences were: $i$ ) sampling of the trajectories was performed at each integration time step, i.e. every $2 \mathrm{fs}$, and $i i) 3$ trajectories of the length of $400 \mathrm{~ns}$ each were produced for a total of $1.2 \mu \mathrm{s}$, for each of the two poly-alanines. The trajectories were then collected in parts of length of 5 ns each, which have been used to calculate the auto- and cross-relaxation functions of the $S_{2}(\Omega)$ functions defined in Eq. 4.

Figs. 9 and 10 compare the calculated rank-2 correlations functions for $\mathbf{2}$ and $\mathbf{8}$, respectively, evaluated via the perturbation treatment described above and superimposed with the same functions obtained from molecular dynamics trajectories. SFB model correlation functions are obtained as the inverse Fourier transforms of the spectral densities (which is the direct output of the model). The correlation functions obtained from the MD trajectories have been corrected to account for the lower viscosity of TIP3P water, which is 2.79 times smaller

than the viscosity of real water at $298 \mathrm{~K} .^{28}$ The comparison is, in our opinion, reasonably good, considering the absence of any fitting procedure or attempt to adjust any parameter of the SFB model, even if some are roughly estimated. For instance no hydrodynamic interactions were employed when calculating the initial free-bead diffusion tensor (see Appendix B of Paper I), and a single effective radius has been assumed for all beads. Quantitative interpretation of relaxation observables (as obtained e.g. from a NMR experiment) based on the SFB approach or augmented versions of the same,${ }^{1}$ will likely require such improvements.

\section{SUMMARY AND CONCLUSIONS}

In this paper we have discussed a first application of the semi-flexible Brownian (SFB) approach, previously introduced in the companion paper $\mathrm{I}^{1}$ from a thorough statistical mechanical description, to treat joint internal and global motion of a molecule, with a focus on the approximate evaluation of spectral densities of orientation correlation functions. The model describes internal motions in the harmonic limit, but accounts for mutual coupling of dynamic units, explicit momenta, and dissipation, all of which are described by a boson bath operator. In particular, we have presented an extended perturbation treatment based on a direct expansion of the kernel operator obtained from a Nakajima-Zwanzig projection, using the properties of the bi-orthonormal eigenfunctions of the (non-Hermitian) bath op- 
erator. This study has been complemented with an alternative efficient approach based on a moment expansion. Two model systems (2 and 8-alanine) have been considered to discuss the computational treatment of the model.

Two goals were set at the beginning of this investigation. ${ }^{1}$ Firstly, we aimed to provide a full systematic description, starting from an atomistic point of view, of the internal/global motion of flexible molecules in solution, based on a direct derivation from the classic Liouville equation of motion, within a clear, general framework. In the process, approximations have been employed to reduce the system complexity and allow for a direct or indirect evaluation of energy and dissipation parameters, but always based on clear physical arguments. The second goal was to provide, at least for a first level of description (SFB model), a fast, efficient way of evaluating spectral densities of interest for the interpretation of nuclear magnetic resonance relaxation experiments. Several developments may be planned for future investigation. The first obvious extension is the evaluation of relaxation times and NOEs observables in solution and in solid state, ${ }^{29}$ which can be accomplished straightforwardly given the availability of spectral densities, at least as obtained from the SFB approach. The latter may be further extended by including large amplitude and concerted motions (e.g. crank-shaft conformational changes): this will require the explicit treatments of a small number of non harmonic internal degrees of freedom, at a modest additional computational cost. Extension to other classes of spectroscopic observables would be also natural, e.g. Site-Directed-Spin-Labeled Electron Spin Resonance, ${ }^{10,11}$ Förster Fluorescence Resonance Energy Transfer. ${ }^{12}$

The importance of rooting a coarse-grained description of macromolecular motions on first principles, cannot, in our opinion, be underestimated. One can learn in the process that a significantly realistic modeling approach can be obtained which retains the main physical aspects, without implying a prohibitive computational cost. For instance, most models usually employed for the interpretation of NMR relaxation data of macromolecules are based on a decoupled internal description of the local dynamics of the probe, e.g. the $\mathrm{N}-\mathrm{H}$ bond in NMR relaxation, thus neglecting possible short, or even, long-range coupling effects. Such an approach, while still providing good agreement with experimental data, may lose important information hidden into fitting parameters. Collective dynamics, that can be for example responsible of allosteric motions in proteins, may not be properly caught by such local models. In our description, on the other hand, it is possible to treat exactly 
even long-range (through bonds and/or through space) couplings, that can originate both from the free energy landscape in internal coordinates or from the hydrodynamic coupling among all the degrees of freedom. To illustrate such a feature of the model introduced in the companion Paper I and here applied to calculate spectral densities of 2-alanine and 8-alanine peptides, we propose in conclusion a very qualitative analysis of the extension and pattern of the couplings of the internal degrees of freedom of 8-alanine. In particular, we focus on the coupling among torsion angles, and as limiting cases two torsion angles are considered as reference to explore the network of couplings. One torsion angle is selected as the first $\psi$ backbone angle, probing the terminal part of the molecule. The second angle is again a $\psi$ angle, located in the middle of the peptidic sequence, between residues 4 and 5 . The effect of coupling due to the energetics of the system is depicted in Figures 11a and $11 \mathrm{~b}$ for the terminal and central $\psi$ angles, respectively. In the figures, the torsion angle taken as reference is colored in cyan. The bonds that are the axes about which a rotation is carried out due to a change in the corresponding torsion angle, are colored with a color map morphing from green to red. The color is related to the magnitude of the elements $a_{i, j}=\log \left|\left(\boldsymbol{\omega}_{K}\right)_{i, j \neq i}\right|$, where $i$ is the index corresponding to the reference torsion angle, while $j$ is the index corresponding to one of the other torsion angles. The color map is defined so that the pure green color corresponds to the smallest $a_{i, j}$, while red to the largest one. Thus, in Figure 11, green indicates small coupling relative to the set of out-of-diagonal elements of $\boldsymbol{\omega}_{K}$ pertinent to the reference torsion angle, dark yellow means medium coupling, while red means high coupling. For the 8-alanine molecule, it is evident a pattern of coupling of this type: a high coupling with $\psi$ torsion angles, medium-low coupling with $\phi$ angles, weak coupling with $\omega$ and lateral chain torsion angles. The pattern shows some larger coupling in the proximity (by bonds or by space) of the reference torsion angle.

Figure 12 shows the same analysis done on the $\boldsymbol{\omega}_{\xi}$ matrix. The way the color map is constructed is the same as described above, with the difference that the quantities used are $b_{i, j}=\log \left|\left(\boldsymbol{\omega}_{\xi}\right)_{i, j \neq i}\right|$. The figure highlights the entity of the hydrodynamic coupling between the reference torsion angle and all the other torsion angles. For both the terminal and center torsion angles, the highest coupling is observed with the central body of the molecule, while it tends to be reduced while approaching the terminal residues. Inspecting Figure 12 it appears that high hydrodynamic coupling is observed mainly with backbone torsion angles, while medium to low coupling is observed with the side chain torsion angles. 
As a final consideration, we would like to point out that the real merit of any modeling approach lies in the capability of rationalizing complex behaviors, which are only apparently caught up by brute force simulations. Among others, we comment here further the seminal modelling approach on polymer dynamics by Perico and Guenza, ${ }^{30,31}$ based on a coarse-grained description of the polymer chain(s), the internal dynamics of which is guided by a harmonic potential energy surface. Autocorrelation functions of a probe segment vector are obtained assuming a Gaussian random process for the dynamics of the Cartesian coordinates of the beads building the polymer and employing the proper Langevin equation. This approach has been also extended to a solution of polymers/macromolecules, deriving the set of generalized Langevin equations (GLE) describing the coupled dynamics of many coarse-grained polymer chains from the complete, classical, Liouville equation in Cartesian coordinates. ${ }^{32,33}$ The GLE-based treatment of the coarse-grained polymers has been also employed to estimate the rescaling of dynamics in coarse-grained simulations. ${ }^{34}$

The key factor in this and other approaches presented in the literature (see I for a larger, but necessarily incomplete, short review) is the inclusion of a treatment as 'exact' as possible of the internal+global dynamics. Our work is based on the same rationale with some main improvements that can be, in our opinion, considered an advancement with respect to previous models. Firstly, our derivation is carried out in internal, natural coordinates. This is not trivial, especially when building the Lagrangian of the complete molecular system, but the benefits are worth the efforts. Working in internal coordinates allows one to reduce more efficiently the complexity of the system with respect to the description in Cartesian coordinates. A second strong point of the computational approach proposed here is the fact that no a priori assumption needs to be done on the shape of the internal free energy landscape. While in the present paper a harmonic internal energy has been chosen for a first level of testing of the methodology and parameterization, the basis is posed for the treatment of non-harmonic generic energy landscapes. To summarize, the whole methodology presented in I and discussed here can be in fact thought as a way to link formally in a rational and integrated way information coming from different sources, to understand the relaxation processes resulting from the mobility of a given molecular structure, interpret and predict spectroscopic observables, and validate parameters evaluated computationally, such as molecular dynamics force-fields and interaction molecular tensors. 


\section{Appendix A: Ornstein-Uhlenbeck operator}

We list here some useful properties of the bi-orthornomal pair of eigenstates of a stochastic Ornstein-Uhlenbeck process described by a linear Fokker-Planck operator. We employ, with some technical changes, the method presented by Leen et al. ${ }^{35}$ We consider a set of $N$ variables, $\mathbf{x}=\left(x_{1}, \ldots, x_{\alpha}, \ldots, x_{N}\right)$, with an equilibrium density probability $p(\mathbf{x})=\exp \left(-x^{2} / 2\right) /(2 \pi)^{N / 2}$. The (symmetrized) time evolution equation for the (symmetrized) conditional probability $p(\mathbf{x}, t)$ is

$$
\frac{\partial p(\mathbf{x}, t)}{\partial t}=-\tilde{\Gamma} p(\mathbf{x}, t)=p(\mathbf{x})^{-1 / 2} \hat{\nabla} \cdot \boldsymbol{\omega}^{\text {io }} p(\mathbf{x}) \cdot \hat{\nabla} p(\mathbf{x})^{-1 / 2} p(\mathbf{x}, t)
$$

here $\hat{\nabla}$ is the gradient operator in $\mathbf{x}, \omega$ is a constant $n \times n$ non-negative square matrix. The adjoint (backward) time evolution operator is $\tilde{\Gamma}^{\dagger}=-p(\mathbf{x})^{-1 / 2} \hat{\nabla} \cdot \boldsymbol{\omega}^{\text {iotr }} p(\mathbf{x}) \cdot \hat{\nabla} p(\mathbf{x})^{-1 / 2}$. The stationary states, $\tilde{\Gamma}|\mathbf{0}\rangle=\tilde{\Gamma}^{\dagger}|\overline{\mathbf{0}}\rangle=0$ are $|\mathbf{0}\rangle=|\overline{\mathbf{0}}\rangle=p(\mathbf{x})^{1 / 2}$. The bi-orthonormal set of eigenstates is defined by $\tilde{\Gamma}|\mathbf{n}\rangle=\Lambda_{\mathbf{n}}|\mathbf{n}\rangle, \tilde{\Gamma}^{\dagger}|\overline{\mathbf{n}}\rangle=\Lambda_{\mathbf{n}}^{*}|\overline{\mathbf{n}}\rangle,\left\langle\overline{\mathbf{n}} \mid \mathbf{n}^{\prime}\right\rangle=\delta_{\mathbf{n}, \mathbf{n}^{\prime}}$, with $\Re \lambda_{\mathbf{n}}>0$ for $\mathbf{n} \neq \mathbf{0}$.

For $\alpha=1, \ldots, N$ we define the vector operators

$$
\hat{\mathbf{S}}^{ \pm}=\mp e^{\mp x^{2} / 4} \hat{\nabla} e^{ \pm x^{2} / 4}
$$

notice that i) $\hat{S}_{\alpha}^{+\dagger}=\hat{S}_{\alpha}^{-}$, ii) $\tilde{\Gamma}=\hat{\mathbf{S}}^{+} \cdot \boldsymbol{\omega}^{\text {io }} \cdot \hat{\mathbf{S}}^{-}$and $\tilde{\Gamma}^{\dagger}=\hat{\mathbf{S}}^{+} \cdot \boldsymbol{\omega}^{\text {iotr }} \cdot \hat{\mathbf{S}}^{-}$. The right and left eigenvectors of the real matrix $\boldsymbol{\omega}$ are: $\boldsymbol{\omega} \mathbf{e}_{\alpha}=\lambda_{\alpha} \mathbf{e}_{\alpha}$ and $\mathbf{f}_{\alpha}^{\operatorname{tr}} \boldsymbol{\omega}=\mathbf{f}_{\alpha}^{\operatorname{tr}} \lambda_{\alpha}$, where $\mathbf{f}_{\alpha}^{\text {tr }} \mathbf{e}_{\beta}=\delta_{\alpha, \beta}$.

Finally, we can now define the operators $\hat{O}_{\alpha}^{+}=\mathbf{e}_{\alpha} \cdot \hat{\mathbf{S}}^{+}$and $\hat{O}_{\alpha}^{-}=\mathbf{f}_{\alpha} \cdot \hat{\mathbf{S}}^{+}$and the functions $f_{\mathbf{n}}=\Pi_{\alpha=1}^{N}\left(\hat{O}_{\alpha}^{+}\right)^{n_{\alpha}}|\mathbf{0}\rangle=\hat{O}^{+}(n)|\mathbf{0}\rangle$ where $\mathbf{n}=\left(n_{1}, \ldots, n_{N}\right)$. The following expressions, which can be obtained by inspection or induction, hold

$$
\begin{aligned}
\tilde{\Gamma} f_{\mathbf{n}} & =\Lambda_{\mathbf{n}} f_{\mathbf{n}}, \quad \Lambda_{\mathbf{n}}=\sum_{\alpha=1}^{n} n_{\alpha} \lambda_{\alpha} \\
{\left[\tilde{\Gamma}, \hat{O}_{\alpha}^{ \pm}\right] } & = \pm \lambda_{\alpha} \hat{O}_{\alpha}^{ \pm} \\
\tilde{\Gamma} \hat{O}_{\alpha}^{ \pm} f_{n} & =\left(\Lambda_{\mathbf{n}} \pm \lambda_{\alpha}\right) \hat{O}_{\alpha}^{ \pm} f_{n}
\end{aligned}
$$

that is $\hat{O}_{\alpha}^{ \pm}$are raising and lowering operators for the set $f_{\mathbf{n}}$, which are eigenfunctions of $\tilde{\Gamma}$ 
with eigenvalues $\Lambda_{\mathbf{n}}$. Analogously, we can find similar expression for the backward operator

$$
\hat{P}_{\alpha}^{+}=\mathbf{f}_{\alpha}^{*} \cdot \hat{\mathbf{S}}^{+}=\left(\hat{O}_{\alpha}^{-}\right)^{\dagger}, \quad \hat{P}_{\alpha}^{-}=\mathbf{e}_{\alpha}^{*} \cdot \hat{\mathbf{S}}^{-}=\left(\hat{O}_{\alpha}^{+}\right)^{\dagger}
$$

Let us now define

$$
g_{\mathbf{n}}=\Pi_{\alpha=1}^{N}\left(\hat{P}_{\alpha}^{+}\right)^{n_{\alpha}}|\mathbf{0}\rangle=\hat{P}^{+}(n)|\mathbf{0}\rangle
$$

and we get

$$
\begin{aligned}
\tilde{\Gamma}^{\dagger} g_{n} & =\Lambda_{\mathbf{n}}^{*} g_{n} \\
{\left[\tilde{\Gamma}^{\dagger}, \hat{P}_{\alpha}^{ \pm}\right] } & = \pm \lambda_{\alpha}^{*} \hat{P}_{\alpha}^{ \pm} \\
\tilde{\Gamma}^{\dagger} \hat{P}_{\alpha}^{ \pm} g_{\mathbf{n}} & =\left(\Lambda_{\mathbf{n}} \pm \lambda_{\alpha}\right)^{*} \hat{P}_{\alpha}^{ \pm} g_{n}
\end{aligned}
$$

stating that $\hat{P}_{\alpha}^{ \pm}$are raising and lowering operators for the set $g_{n}$, with eigenvalues $\Lambda_{\mathbf{n}}^{*}$. Also, we can show that

$$
\begin{aligned}
{\left[\hat{O}_{\alpha}^{-}, \hat{O}_{\beta}^{-}\right] } & =\left[\hat{O}_{\alpha}^{+}, \hat{O}_{\beta}^{+}\right]=0 \\
{\left[\hat{O}_{\alpha}^{-}, \hat{O}_{\beta}^{+}\right] } & =\delta_{\alpha, \beta} \\
\hat{P}_{\alpha}^{ \pm} & =\left(\hat{O}^{ \pm}\right)^{\dagger}
\end{aligned}
$$

Finally, we can show from Eqs. A7 that $f_{\mathbf{n}}, g_{\mathbf{n}}$ form a bi-orthogonal set

$$
\left\langle g_{\mathbf{n}} \mid f_{\mathbf{n}^{\prime}}\right\rangle=\left(\Pi_{\alpha=1}^{N} n_{\alpha} !\right) \delta_{\mathbf{n n}^{\prime}}
$$

For sake of convenience, we define explicitly the bi-orthonormal set and the effect of applying raising and lowering operators

$$
\begin{aligned}
|\mathbf{n}\rangle & =\left(\Pi_{\alpha=1}^{N} n_{\alpha} !\right)^{-1 / 2} f_{\mathbf{n}} \\
|\overline{\mathbf{n}}\rangle & =\left(\Pi_{\alpha=1}^{N} n_{\alpha} !\right)^{-1 / 2} g_{\mathbf{n}} \\
\left(\hat{O}_{\beta}^{-}\right)^{m}|\mathbf{n}\rangle & =\left[\frac{n_{\beta} !}{\left(n_{\beta}-m\right) !}\right]^{1 / 2}\left|n_{1}, \ldots, n_{\beta}-m, \ldots, n_{N}\right\rangle \\
\left(\hat{O}_{\beta}^{+}\right)^{m}|\mathbf{n}\rangle & =\left[\frac{\left(n_{\beta}+m\right) !}{n_{\beta} !}\right]^{1 / 2}\left|n_{1}, \ldots, n_{\beta}+m, \ldots, n_{N}\right\rangle
\end{aligned}
$$

where $|\mathbf{0}\rangle=|\overline{\mathbf{0}}\rangle=p(\mathbf{x})^{1 / 2} ;\left(\hat{O}_{\beta}^{-}\right)^{m}|\mathbf{n}\rangle=0$ for $m>n_{\beta}$. 


\section{Appendix B: TTOC expansion}

We want to obtain 18. We define $\mathbf{E}=\left(\mathbf{e}_{1}, \ldots, \mathbf{e}_{N}\right), \mathbf{F}=\left(\mathbf{f}_{1}, \ldots, \mathbf{f}_{N}\right)^{\text {tr }}$; notice that $\hat{\mathbf{O}}^{+}=\mathbf{E}^{\operatorname{tr}} \hat{\mathbf{S}}^{+}, \hat{\mathbf{O}}^{-}=\mathbf{F} \hat{\mathbf{S}}^{-}$and, since $\mathbf{F E}=\mathbf{E F}=\mathbf{1}, \hat{\mathbf{S}}^{+}=\mathbf{F}^{\operatorname{tr}} \hat{\mathbf{O}}^{+}, \hat{\mathbf{S}}^{-}=\mathbf{E} \hat{\mathbf{O}}^{-}$. We can write

$$
\tilde{\Gamma}_{\mathrm{int}}=\hat{\mathbf{M}} \cdot\left(\boldsymbol{\omega}^{+} \hat{\mathbf{O}}^{+}+\boldsymbol{\omega}^{-} \hat{\mathbf{O}}^{-}\right)
$$

where $\boldsymbol{\omega}^{+}=\boldsymbol{\omega}^{\text {int }} \mathbf{F}^{\text {tr }}, \boldsymbol{\omega}^{-}=\boldsymbol{\omega}^{\text {int }} \mathbf{E}$. It is useful to recover by inspection the first terms of Eq. 18. For sake of simplicity, we shall call $\mathcal{E}_{\nu}$ the subset of eigenfunctions of $\tilde{\Gamma}$ with $\sum_{\alpha=1}^{N} n_{\alpha}=\nu$ (for $\nu=0$ the subset contains only $|\mathbf{0}\rangle$, for $\nu=1$ all the 'first excited' states and so on). One can see that the $k=0$ term is null: $\left\langle\overline{\mathbf{0}}\left|\tilde{\Gamma}_{\text {int }}\right| \mathbf{0}\right\rangle=\left\langle\overline{\mathbf{0}}\left|\hat{\mathbf{M}} \cdot\left(\boldsymbol{\omega}^{+} \hat{\mathbf{O}}^{+}+\boldsymbol{\omega}^{-} \hat{\mathbf{O}}^{-}\right)\right| \mathbf{0}\right\rangle=0$, since $\hat{\mathrm{O}}^{+}$applied to $|\mathbf{0}\rangle$ generates only elements of $\mathcal{E}_{1}$, therefore orthogonal to $\langle\overline{\mathbf{0}}|$, and $\hat{\mathbf{O}}^{-}|\mathbf{0}\rangle=0$. The term $k=1$ is obtained as

$$
\begin{aligned}
g_{1}(\omega) & =-\left\langle\overline{\mathbf{0}}\left|\tilde{\Gamma}_{\text {int }}\left(i \omega+\tilde{\Gamma}_{0}\right)^{-1} \tilde{Q} \tilde{\Gamma}_{\text {int }}\right| \mathbf{0}\right\rangle=-\sum_{\mathbf{n} \neq \mathbf{0}}\left\langle\overline{\mathbf{0}}\left|\tilde{\Gamma}_{\text {int }}\right| \mathbf{n}\right\rangle\left(i \omega+\Omega_{n}\right)^{-1}\left\langle\overline{\mathbf{n}}\left|\tilde{\Gamma}_{\text {int }}\right| \mathbf{0}\right\rangle \\
& =-\sum_{p q} \sum_{\alpha=1}^{N} \frac{\omega_{p \alpha}^{-} \omega_{q \alpha}^{+}}{i \omega+\lambda_{\alpha}} \hat{M}_{p} \hat{M}_{q}
\end{aligned}
$$

where the last expression is obtained using the properties of $\hat{\mathbf{O}}^{ \pm}$. It follows that the generalized diffusion tensor ${ }^{[2]} D(\omega)$ has components

$$
{ }^{[2]} D_{p q}(\omega)=\sum_{\alpha=1}^{N} \frac{\omega_{p \alpha}^{-} \omega_{q \alpha}^{+}}{i \omega+\lambda_{\alpha}}
$$

We now generalize the derivation above to obtain a close expression for the generic $\hat{g}_{k}(\omega)$. We see first that for $k$ even, all terms go to zero. In fact we can write

$$
\hat{g}_{2 l}(\omega)=\sum_{\mathbf{n}_{1}, \mathbf{n}_{2}, \ldots, \mathbf{n}_{2 l}}\left[\prod_{r=1, \ldots, 2 l}\left(i \omega+\Lambda_{\mathbf{n}_{\alpha}}\right)^{-1}\right]\left\langle\overline{\mathbf{0}}\left|\tilde{\Gamma}_{\mathrm{int}}\right| \mathbf{n}_{1}\right\rangle\left\langle\overline{\mathbf{n}}_{1}\left|\tilde{Q} \tilde{\Gamma}_{\mathrm{int}}\right| \mathbf{n}_{2}\right\rangle \ldots\left\langle\overline{\mathbf{n}}_{2 l}\left|\tilde{Q} \tilde{\Gamma}_{\mathrm{int}}\right| \mathbf{0}\right\rangle
$$

let us consider the first matrix element of $\tilde{\Gamma}_{\text {int }}$ on the left: it is not zero only for $\left|\mathbf{n}_{1}\right\rangle \in \mathcal{E}_{1}$, which implies that the next one is not zero only if $\left|\mathbf{n}_{2}\right\rangle \in \mathcal{E}_{0}, \mathcal{E}_{2}$ and so on, showing that in the end $\left|\mathbf{n}_{2 l}\right\rangle \in \mathcal{E}_{0}, \ldots, \mathcal{E}_{2 l}$ in order to have all terms surviving but the last one on the right; hence $\left\langle\overline{\mathbf{n}}_{2 l}\left|\tilde{Q} \tilde{\Gamma}_{\text {int }}\right| \mathbf{0}\right\rangle=0$ since $\left|\mathbf{n}_{2 l}\right\rangle \notin \mathcal{E}_{1}$. Finally, we evaluate $\hat{g}_{2 l+1}(\omega)$. Following an 
analogous procedure, one sees that the generic form of $\hat{g}_{2 l+1}(\omega)$ must be

$$
\hat{g}_{2 l+1}(\omega)=-\sum_{p_{1}, \ldots, p_{2(l+1)}}[2(l+1)] D_{p_{1} \ldots p_{2(l+1)}}(\omega) \hat{M}_{p_{1}} \ldots \hat{M}_{p_{2(l+1)}}
$$

since each term in $\tilde{\Gamma}_{\text {int }}$ is linear in the components of $\hat{\mathbf{M}}$ and each terms in $\tilde{\Gamma}_{0}$ generates a constants factor.

To evaluate explicitly ${ }^{[2(l+1)]} D_{p_{1} \ldots p_{2(l+1)}}(\omega)$ we define first the ancillary coefficients, function of two generic $N$-tuples $\mathbf{m}, \mathbf{n}$ :

$$
c_{p}(\mathbf{m}, \mathbf{n})=\sum_{\alpha=1}^{N} \omega_{p \alpha}^{+}\left(n_{\alpha}+1\right)^{1 / 2} \delta_{\mathbf{m}, \mathbf{n}+1_{\alpha}}+\omega_{p \alpha}^{-}\left(n_{\alpha}\right)^{1 / 2} \delta_{\mathbf{m}, \mathbf{n}-1_{\alpha}}
$$

where $\mathbf{n}+1_{\alpha}$ is the $N$-tuple obtain by $\mathbf{n}$ substituting $n_{\alpha}$ with $n_{\alpha}+1$; we also generalize slightly the definitions $\hat{g}_{2 l+1}(\omega) \equiv \hat{g}_{2 l+1}(\omega, 0,0)$ evidencing the fact they are matrix elements to $\langle\overline{\mathbf{0}}|$ and $|\mathbf{0}\rangle$; analogously ${ }^{[2(l+1)]} D_{p_{1} \ldots p_{2(l+1)}}(\omega) \equiv{ }^{[2(l+1)]} D_{p_{1} \ldots p_{2(l+1)}}(\omega, 0,0)$. We can now write:

$$
\begin{aligned}
\hat{g}_{2 l+1}(\omega, \mathbf{m}, \mathbf{n}) & =-\left\langle\overline{\mathbf{m}}\left|\tilde{\Gamma}_{\mathrm{int}}\left[\left(i \omega+\tilde{\Gamma}_{0}\right)^{-1} \tilde{Q}_{\Gamma_{\mathrm{int}}}\right]^{2 l+1}\right| \mathbf{n}\right\rangle \\
& =-\sum_{\mathbf{m}^{\prime}}\left\langle\overline{\mathbf{m}}\left|\tilde{\Gamma}_{\mathrm{int}}\left[\left(i \omega+\tilde{\Gamma}_{0}\right)^{-1} \tilde{Q} \tilde{\Gamma}_{\mathrm{int}}\right]^{2 l-1}\right| \mathbf{m}^{\prime}\right\rangle\left\langle\overline{\mathbf{m}}^{\prime}\left|\left[\left(i \omega+\tilde{\Gamma}_{0}\right)^{-1} \tilde{Q} \tilde{\Gamma}_{\mathrm{int}}\right]^{2}\right| \mathbf{n}\right\rangle \\
& =-\sum_{\mathbf{m}^{\prime}} \hat{g}_{2 l-1}\left(\omega, \mathbf{m}, \mathbf{m}^{\prime}\right)\left\langle\overline{\mathbf{m}}^{\prime}\left|\left[\left(i \omega+\tilde{\Gamma}_{0}\right)^{-1} \tilde{Q}_{\Gamma_{\mathrm{int}}}\right]^{2}\right| \mathbf{n}\right\rangle
\end{aligned}
$$

but we can see that

$$
\left\langle\overline{\mathbf{m}}^{\prime}\left|\left[\left(i \omega+\tilde{\Gamma}_{0}\right)^{-1} \tilde{Q} \tilde{\Gamma}_{\mathrm{int}}\right]^{2}\right| \mathbf{n}\right\rangle \sum_{\mathbf{m}^{\prime \prime}}\left(i \omega+\Lambda_{\mathbf{m}}^{\prime}\right)^{-1}\left(i \omega+\Lambda_{\mathbf{m}^{\prime \prime}}\right)^{-1} \sum_{q_{1}, q_{2}} c_{q_{1}}\left(\mathbf{m}^{\prime}, \mathbf{m}^{\prime \prime}\right) c_{q_{2}}\left(\mathbf{m}^{\prime \prime}, \mathbf{n}\right) \hat{M}_{q_{1}} \hat{M}_{q_{2}}
$$

It follows that

$$
\begin{aligned}
\hat{g}_{2 l+1}(\omega, \mathbf{m}, \mathbf{n}) & =\sum_{p_{1}, \ldots, p_{2(l+1)}}{ }^{[2(l+1)]} D_{p_{1} \ldots p_{2(l+1)}}(\omega, \mathbf{m}, \mathbf{n}) \hat{M}_{p_{1}} \ldots \hat{M}_{p_{2(l+1)}} \\
& =\sum_{p_{1}, \ldots, p_{2 l}} \sum_{\mathbf{m}^{\prime} \mathbf{m}^{\prime \prime}}{ }^{[2 l]} D_{p_{1} \ldots p_{2 l}}\left(\omega, 0, m^{\prime}\right) \hat{M}_{p_{1}} \ldots \hat{M}_{p_{2 l}} \\
& \times\left(i \omega+\Lambda_{\mathbf{m}^{\prime}}\right)^{-1}\left(i \omega+\Lambda_{\mathbf{m}^{\prime \prime}}\right)^{-1} \sum_{q_{1}, q_{2}} c_{q_{1}}\left(\mathbf{m}^{\prime}, \mathbf{m}^{\prime \prime}\right) c_{q_{2}}\left(\mathbf{m}^{\prime \prime}, \mathbf{n}\right) \hat{M}_{q_{1}} \hat{M}_{q_{2}}
\end{aligned}
$$


therefore

$$
{ }^{[2(l+1)]} D_{p_{1} \ldots p_{2(l+1)}}(\omega, 0, \mathbf{n})=\sum_{\mathbf{m}^{\prime} \mathbf{m}^{\prime \prime}}{ }^{[2 l]} D_{p_{1} \ldots p_{2 l}}\left(\omega, 0, \mathbf{m}^{\prime}\right) \frac{c_{p_{2 l+1}}\left(\mathbf{m}^{\prime}, \mathbf{m}^{\prime \prime}\right) c_{p_{2(l+1)}}\left(\mathbf{m}^{\prime \prime}, \mathbf{n}\right)}{\left(i \omega+\Lambda_{\mathbf{m}^{\prime}}\right)\left(i \omega+\Lambda_{\mathbf{m}^{\prime \prime}}\right)}
$$

which is recursive expression for ${ }^{[2(l+1)]} D_{p_{1} \ldots p_{2(l+1)}}(\omega, 0, n)$; for $l=0$ we have the generalized equivalent of Eq. B3

$$
{ }^{[2]} D_{p q}(\omega, 0, \mathbf{n})=\sum_{\mathbf{m}} \frac{c_{p_{1}}(0, \mathbf{m}) c_{p_{2}}(\mathbf{m}, \mathbf{n})}{i \omega+\Lambda_{\mathbf{m}}}
$$

and finally, by inspection, with $\mathbf{n}=\mathbf{0}$ we can find the closed expression

$$
{ }^{[2 l]} D_{p_{1} \ldots p_{2 l}}(\omega)=\sum_{\mathbf{m}_{1}, \ldots, \mathbf{m}_{2 l-1}} \frac{c_{p_{1}}\left(0, \mathbf{m}_{1}\right) c_{p_{2}}\left(\mathbf{m}_{1}, \mathbf{m}_{2}\right) \ldots c_{p_{2 l-1}}\left(\mathbf{m}_{2 l-2}, \mathbf{m}_{2 l-1}\right) c_{p_{2 l}}\left(\mathbf{m}_{2 l-1}, 0\right)}{\left(i \omega+\Lambda_{\mathbf{m}_{1}}\right)\left(i \omega+\Lambda_{\mathbf{m}_{2}}\right) \ldots\left(i \omega+\Lambda_{\mathbf{m}_{2 l-1}}\right)}
$$

\section{Appendix C: Evaluation of spectral densities from the moments}

We define scaled and rotated moments, using the matrix of right eigenvectors of $\boldsymbol{\omega}$.

$$
\begin{aligned}
& M_{l}^{(0)}=m_{l}^{(0)} \\
& \mathbf{M}_{l}^{(1)}=\mathbf{E m}_{l}^{(1)} \\
& \mathbf{M}_{l}^{(2}=\left(m_{l}^{(0)} \mathbf{1}+\mathbf{E m}_{l}^{(2)} \mathbf{E}^{\mathrm{tr}}\right)
\end{aligned}
$$

initial conditions are now $m_{l}^{(0)}=\delta_{l \bar{l}}, \mathbf{m}_{l}^{(1)}=\mathbf{0}, \mathbf{m}_{l}^{(2)}=\mathbf{0}$, and Eqs. IV take the form

$$
\begin{aligned}
& \dot{m}_{l}^{(0)}=-\sum_{l^{\prime}} e_{l l^{\prime}}^{\mathrm{tr}} \mathbf{E m}_{l^{\prime}}^{(1)} \\
& \dot{\mathbf{m}}_{l}^{(1)}=-\boldsymbol{\lambda} \mathbf{m}_{l}^{(1)}-\sum_{l^{\prime}}\left(m_{l^{\prime}}^{(0)} \mathbf{S}+\mathbf{m}_{l^{\prime}}^{(2)} e_{l l^{\prime}}\right) \\
& \dot{\mathbf{m}}_{l}^{(2)}=-\dot{m}_{l}^{(0)} \mathbf{S}-\left(\boldsymbol{\lambda} \mathbf{m}_{l}^{(2)}+\mathbf{m}_{l}^{(2)} \boldsymbol{\lambda}\right)
\end{aligned}
$$

with the definitions of vectors $\left(\mathbf{e}_{l l^{\prime}}\right)_{j}=e_{j, l l^{\prime}}=\sum_{j} E_{i j} \bar{\omega}_{i, l l^{\prime}}^{\text {int }}$ and matrix $\mathbf{S}=\mathbf{F F}^{\mathrm{tr}}$. The advantage of the new formulation is that the subset of equations in the second order moments, which has the form of a differential Lyapunov equation, is now uncoupled, since $\boldsymbol{\lambda}$, the matrix of eigenvalues of $\boldsymbol{\omega}$ io, is diagonal. Taking the Fourier-Laplace transform of Eqs. C2 we 
get for the second order scaled moments

$$
\tilde{m}_{l, i j}^{(2)}=-\frac{S_{i j}\left(i \omega \tilde{m}_{l}^{(0)}-m_{l}^{(0)}\right)}{i \omega+\lambda_{i}+\lambda_{j}}
$$

for the first order scaled moments

$$
\tilde{m}_{l, i}^{(1)}=-\sum_{l^{\prime} j} \frac{S_{i j}\left[\left(\lambda_{i}+\lambda_{j}\right) \tilde{m}_{l^{\prime}}^{(0)}+m_{l^{\prime}}^{(0)}(0)\right]}{\left(i \omega+\lambda_{i}\right)\left(i \omega+\lambda_{i}+\lambda_{j}\right)} e_{j, l l^{\prime}}
$$

and for the zero order scaled moments

$$
i \omega \tilde{m}_{l}^{(0)}-m_{l}^{(0)}(0)=\sum_{l^{\prime} l^{\prime \prime} i j} e_{i, l l^{\prime}} \frac{S_{i j}\left[\left(\lambda_{i}+\lambda_{j}\right) \tilde{m}_{l^{\prime \prime}}^{(0)}+m_{l^{\prime \prime}}^{(0)}(0)\right]}{\left(i \omega+\lambda_{i}\right)\left(i \omega+\lambda_{i}+\lambda_{j}\right)} e_{j, l^{\prime} l^{\prime \prime}}
$$

where the tilde indicates a Fourier-Laplace transform. Taking into account the initial conditions and rearranging one gets Eq. 31.

\section{Appendix D: Miscellaneous relations}

\section{J's and j's}

We consider here the normalized real combinations of Wigner functions of rank $L$ :

$$
S_{l}^{L}(\Omega)=\sqrt{\frac{2 L+1}{8 \pi^{2}}} \times\left\{\begin{array}{cc}
\frac{1}{\sqrt{2}}\left[\mathcal{D}_{0, l}^{L}(\Omega)+(-1)^{l} \mathcal{D}_{0,-l}^{L}(\Omega)\right] & 0<l \leq L \\
\mathcal{D}_{0,0}^{L}(\Omega) & l=0 \\
\frac{i}{\sqrt{2}}\left[\mathcal{D}_{0, l}^{L}(\Omega)-(-1)^{l} \mathcal{D}_{0,-l}^{L}(\Omega)\right] & -L \leq l<0
\end{array}\right.
$$

in the main text only second $\operatorname{rank}(L=2)$ functions have been considered, $S_{l} \equiv S_{l}^{2}$. The $S_{l}^{L}$ can be written in a more compact form as

$$
S_{l}^{L}=\sum_{k} T_{l k} \sqrt{\frac{2 L+1}{8 \pi^{2}}} \mathcal{D}_{0, l}^{L}(\Omega)
$$


and the unitary matrix $\mathbf{T}$ is defined as

$$
\mathbf{T}=\frac{1}{\sqrt{2}}\left(\begin{array}{ccccccc}
i & 0 & \ldots & 0 & \ldots & 0 & -i(-1)^{L} \\
i & 0 & \ldots & 0 & \ldots & 0 & -i(-1)^{L-1} \\
0 & 0 & \ldots & \sqrt{2} & \ldots & 0 & 0 \\
(-1)^{-L+1} & 0 & \ldots & 0 & \ldots & 0 & 1 \\
(-1)^{-L} & 0 & \ldots & 0 & \ldots & 0 & 1
\end{array}\right)
$$

The spectral densities $J_{l, \bar{l}}^{L}(\omega)$ are defined

$$
J_{l \bar{l}}(\omega)=(2 L+1)\left\langle\mathcal{D}_{0, l}^{L}(\Omega)^{*}\left|(i \omega+\hat{\Gamma})^{-1}\right| \mathcal{D}_{0, \bar{l}}^{L}(\Omega) \rho\right\rangle_{\mathcal{Q}}
$$

while the 'little' spectral densities $j_{l, \bar{l}}^{L}(\omega)$ are

$$
j_{l \bar{l}}^{L}(\omega)=8 \pi^{2}\left\langle S_{l}^{L}\left|(i \omega+\hat{\Gamma})^{-1}\right| S_{\bar{l}}^{L} \rho\right\rangle_{\mathcal{Q}}
$$

The relations between the two sets for a given $\omega$ are listed in Table V

TABLE V. Relations between $J_{l, \bar{l}}^{L}$ and $j_{l, \bar{l}}^{L}$

\begin{tabular}{c|ccc}
$J_{l, \bar{l}}^{L}$ & $\bar{l}<0$ & $\bar{l}=0$ & $\bar{l}>0$ \\
\hline$l<0$ & $\frac{1}{2}\left[j_{l \bar{l}}-(-1)^{l} j_{-l \bar{l}}-(-1)^{\bar{l}} j_{l-\bar{l}}+(-1)^{l+\bar{l}} j_{-l-\bar{l}}\right]$ & $-\frac{i}{\sqrt{2}}\left[j_{l 0}-(-1)^{l} j_{-l 0}\right]$ & $-\frac{i}{2}\left[j_{l \bar{l}}-(-1)^{l} j_{-l \bar{l}}+(-1)^{\bar{l}} j_{l-\bar{l}}-(-1)^{l+\bar{l}} j_{-l-\bar{l}}\right]$ \\
$l=0$ & $\frac{i}{\sqrt{2}}\left[j_{0 \bar{l}}-(-1)^{\bar{l}} j_{0-\bar{l}}\right]$ & $j_{00}$ & $\frac{1}{\sqrt{2}}\left[j_{0 \bar{l}}+(-1)^{\bar{l}} j_{0-\bar{l}}\right]$ \\
$l>0$ & $\frac{i}{2}\left[j_{l \bar{l}}+(-1)^{l} j_{-l \bar{l}}-(-1)^{\bar{l}} j_{l-\bar{l}}-(-1)^{l+\bar{l}_{j}} j_{-l-\bar{l}}\right]$ & $\frac{1}{\sqrt{2}}\left[j_{l 0}+(-1)^{l} j_{-l 0}\right]$ & $\frac{1}{2}\left[j_{l \bar{l}}+(-1)^{l} j_{-l \bar{l}}+(-1)^{\bar{l}} j_{l-\bar{l}}+(-1)^{l+\bar{l}_{j}} j_{-l-\bar{l}}\right]$ \\
\hline
\end{tabular}

\section{Matrix elements of $\hat{M}_{p}$}

We calculate here matrix elements ${ }^{S} M_{p, l l^{\prime}}^{L}=\left\langle S_{l}^{L}\left|\hat{M}_{p}\right| S_{l^{\prime}}^{L}\right\rangle_{\Omega}$. First we evaluate from standard expressions the matrix elements with respect to the (normalized) Wigner matrix elements $M_{p, l \bar{l}}^{L}=(2 L+1)\left\langle\left|\hat{M}_{p}\right|\right\rangle_{\Omega} / 8 \pi^{2}$ :

$$
\begin{aligned}
& M_{1, l \bar{l}}^{L}=-i\left(c_{L, \bar{l}}^{-} \delta_{l \bar{l}-1}+c_{L, \bar{l}}^{+} \delta_{l \bar{l}+1}\right) \\
& M_{2, l \bar{l}}^{L}=-\left(c_{L, \bar{l}}^{-} \delta_{l \bar{l}-1}-c_{L, \bar{l}}^{+} \delta_{l \bar{l}+1}\right) \\
& M_{3, l \bar{l}}^{L}=-i \bar{l} \delta_{l \bar{l}}
\end{aligned}
$$


where $c_{L, l}^{ \pm}=\sqrt{L(L+1)-l(l \pm 1)}$ and therefore

$$
{ }^{S} M_{p, l \bar{l}}^{L}=\sum_{k \bar{k}} T_{l k}^{*} M_{p, k \bar{k}}^{L} T_{\overline{l k}}
$$

\section{Matrix A}

Next we evaluate the matrix elements $\mathbf{A}$. The block structure of matrix $\mathbf{A}$ is

$$
\mathbf{A}=\left(\begin{array}{ccc}
\mathbf{0} & \mathbf{A}_{01} & \mathbf{0} \\
\mathbf{0} & \mathbf{A}_{11} & \mathbf{A}_{12} \\
\mathbf{A}_{20} & \mathbf{0} & \mathbf{A}_{22}
\end{array}\right)
$$

where the first diagonal block has dimensions $(2 L+1) \times(2 L+1)$, the second diagonal block $(2 L+1) N \times(2 L+1) N$, the third diagonal block $(2 L+1) N^{2} \times(2 L+1) N^{2}$. The overall dimensions of $\mathbf{A}$ are $(2 L+1)\left(1+N+N^{2}\right) \times(2 L+1)\left(1+N+N^{2}\right)$. Explicit non-null matrix elements are defined as follows

$$
\begin{aligned}
\left(\mathbf{A}_{01}\right)_{l, l^{\prime} j} & =-e_{j, l l^{\prime}} \\
\left(\mathbf{A}_{11}\right)_{l i, l^{\prime} j} & =-\lambda_{i} \delta_{i j} \delta_{l l^{\prime}} \\
\left(\mathbf{A}_{12}\right)_{l i, l^{\prime} j k} & =-e_{k, l l^{\prime}} \delta_{i j} \\
\left(\mathbf{A}_{20}\right)_{l i j, l^{\prime}} & =\left[\mathbf{F}\left(\boldsymbol{\omega}^{\mathrm{io}}+\boldsymbol{\omega}^{\mathrm{iotr}}\right) \mathbf{F}^{\mathrm{tr}}\right]_{i j} \delta_{l l^{\prime}}=(\boldsymbol{\lambda} \mathbf{S}+\mathbf{S} \boldsymbol{\lambda})_{i j} \delta_{l l^{\prime}} \\
\left(\mathbf{A}_{22}\right)_{l i j, l^{\prime} k p} & =-\left(\lambda_{i}+\lambda_{j}\right) \delta_{i k} \delta_{j p} \delta_{l l^{\prime}}
\end{aligned}
$$

where indexes $l, l^{\prime}$ run from $-L$ to $L, i, j, k, p$ from 1 to $N$.

\section{Appendix E: Parametrization of SFB model and MD simulations}

As briefly reported in the Results and Discussion Section, the parametrization of the SFB model followed this protocol. First, the molecule ( $\mathbf{2}$ or $\mathbf{8})$ was minimized in vacuo using the MMTK software ${ }^{26}$ and the Amber99 force field. ${ }^{25}$ The software produced in house, called SALEM, that implements the SFB model is programmed to automatically carry out the energy minimization once a PDB file is provided in input. SALEM, will be available as soon as it will be made user-friendly. MMTK is also employed to compute the Hessian of 
TABLE VI. Setup conditions for the new MD simulations of $\mathbf{2}$ and $\mathbf{8}$ employed for the calculation of the correlation functions

\begin{tabular}{ll}
\hline Parameter & Value \\
\hline Cubic box side length $/ \AA$ & 30 \\
Boundary conditions & periodic \\
Number of water molecules $^{a}$ & 900,853 \\
Force field & CHARMM22 with CMAP correction for the \\
& peptides, ${ }^{36}$ TIP3P for water \\
Ensemble & NpT \\
Thermostat & Langevin, $T=298.15 \mathrm{~K}$ \\
Barostat & Langevin, piston period 200 fs, piston decay \\
& 100 fs, $p=1$ atm \\
Non-bonded interactions cut-off $/ \AA^{b}$ & 12.0 \\
Electrostatics & particle mesh Ewald, order 6, tolerance $10^{-5}$ \\
Integration time step $/$ fs $^{c}$ & 2 \\
Dumping frequency & each 1 MD step \\
Minimization steps & 5000 \\
Heating time $/$ ps & 72 \\
Equilibration time $/ \mathrm{ns}_{\text {Production time } / \mathrm{ns}}$ & 2 \\
\hline
\end{tabular}

${ }^{a}$ For $(\mathrm{ALA}-)_{2}$ and $(\mathrm{ALA}-)_{8}$ : number of molecules, chosen to reproduce the density of bulk water at $298.15 \mathrm{~K}$.

${ }^{b}$ Switching at $10.0 \AA$; pairlist set equal to $13.5 \AA$.

${ }^{c}$ All bonds with $\mathrm{H}$ atoms were constrained with the SHAKE algorithm.

the energy in Cartesian coordinates. The latter is then converted into internal coordinates analytically. ${ }^{27}$ The first three backbone atoms have been selected to build the reference molecular frame. For the calculation of the friction tensor, these parameters have been chosen temperature $T=298.15 \mathrm{~K}$, viscosity $\eta=0.894 \mathrm{cP}$, stick boundary conditions, and an effective radius $R_{e}=0.5 \AA$ for the atoms, which is a 'standard' value when computing the friction tensor omitting the hydrodynamic interactions between the beads, as it is actually implemented in SALEM.

New MD trajectories have been computed to calculate the correlation functions reported in Figs. 9 and 10. For each of the two molecules, $3 \times 400$ ns-long MD simulations have been carried out with NAMD, ${ }^{37}$ following a standard protocol consisting in energy minimization, heating, equilibration, and production. Parameters and conditions for the MD simulations are reported in Table VI. 


\section{ACKNOWLEDGMENTS}

The authors thank Fondazione della Cassa di Risparmio di Padova e Rovigo (Project: Modeling and Monitoring Motions in Proteins- $\mathrm{M}_{3} \mathrm{PC}$ ) and the Italian Ministry of Education, of University and of Research (Project: Prin 2012T9XHH7). Computational work has been carried out on the C3P (Computational Chemistry Community in Padua) HPC facility of the Department of Chemical Sciences of the University of Padua.

\section{REFERENCES}

${ }^{1}$ A. Polimeno, M. Zerbetto, and D. Abergel, "Stochastic modeling of macromolecules in solution. i. relaxation processes," (2019), submitted.

${ }^{2}$ G. J. Moro, "A stochastic model for crankshaft transitions," J. Phys. Chem. 100, 16419$16422(1996)$.

${ }^{3}$ B. Nigro and G. J. Moro, "A stochastic model for crankshaft transitions. ii. analysis of transition dynamics," J. Phys. Chem. B 106, 7365-7375 (2002).

${ }^{4}$ H. Risken, The Fokker-Planck Equation: Methods of Solution and Applications (SpringerVerlag, 1984).

${ }^{5}$ J. Cavanagh, W. J. Fairbrother, I. A. G. Palmer, N. J. Skelton, and M. Rance, Protein NMR Spectroscopy: Principles and Practice (Elsevier Science, 2010).

${ }^{6}$ K. Loth, P. Pelupessy, and G. Bodenhausen, "Chemical shift anisotropy tensors of carbonyl, nitrogen, and amide proton nuclei in proteins through cross-correlated relaxation in nmr spectroscopy," J. Am. Chem. Soc. 127, 6062-6068 (2005).

${ }^{7}$ D. Sheppard, D.-W. Li, R. Godoy-Ruiz, R. Brueschweiler, and V. Tugarinov, "Variation in quadrupole couplings of a deuterons in ubiquitin suggests the presence of c-alpha-h-alpha center dot center dot center dot $\mathrm{o}=\mathrm{c}$ hydrogen bonds," J. Am. Chem. Soc. 132, 7709-7719 (2010).

${ }^{8}$ E. Bucci and R. F. Steiner, "Anisotropy decay of fluorescence as an experimental approach to protein dynamics," Biophys. Chem. 30, 199-224 (1988).

${ }^{9}$ B. Vergani, M. Kintrup, W. Hillen, H. Lami, E. Piemont, E. Bombarda, P. Alberti, S. M. Doglia, and M. Chabbert, "Backbone dynamics of tet repressor alpha 8 boolean and alpha 9 loop," Biochemistry-US 39, 2759-2768 (2000). 
${ }^{10}$ W. L. Hubbell, H. S. Mchaourab, C. Altenbach, and M. A. Lietzow, "Watching proteins move using site-directed spin labeling," Structure 4, 779-783 (1996).

${ }^{11}$ Z. Zhang, M. R. Fleissner, D. S. Tipikin, Z. Liang, J. K. Moscicki, K. A. Earle, W. L. Hubbell, and J. H. Freed, "Multifrequency electron spin resonance study of the dynamics of spin labeled t4 lysozyme," J. Phys. Chem. B 114, 5503-5521 (2010).

${ }^{12}$ H. Hofmann, F. Hillger, S. H. Pfeil, A. Hoffmann, D. Streich, D. Haenni, D. Nettels, E. A. Lipman, and B. Schuler, "Single-molecule spectroscopy of protein folding in a chaperonin cage," P. Natl. Acad. Sci. USA 107, 11793-11798 (2010).

${ }^{13}$ L. Hamon, D. Pastre, P. Dupaigne, C. L. Breton, E. L. Cam, and O. Pietrement, "Highresolution afm imaging of single-stranded dna-binding (ssb) protein-dna complexes," Nucleic Acids Res. 35, e58 (2007).

${ }^{14}$ R. Zwanzig, "On the identity of three generalized master equations," Physica 30, 1109-1123 (1964).

${ }^{15}$ R. Zwanzig, "Nonlinear generalized langevin equations," J. Stat. Phys. 9, 215-220 (1973).

${ }^{16}$ R. N. Zare, Angular momentum: understanding spatial aspects in chemistry and physics (Wiley, 1988).

${ }^{17}$ A. Montoya-Castillo and D. R. Reichman, "Approximate but accurate quantum dynamics from the Mori formalism: I. Nonequilibrium dynamics," J. Chem. Phys. 144 (2016), $10.1063 / 1.4948408$.

${ }^{18}$ Z. Gong, Z. Tang, S. Mukamel, J. Cao, and J. Wu, "A continued fraction resummation form of bath relaxation effect in the spin-boson model," J. Chem. Phys. 142 (2015), 10.1063/1.4913198.

${ }^{19}$ M. G. Mavros and T. Van Voorhis, "Resummed memory kernels in generalized system-bath master equations," J. Chem. Phys. 141 (2014), 10.1063/1.4891669.

${ }^{20}$ Q. Shi and E. Geva, "A new approach to calculating the memory kernel of the generalized quantum master equation for an arbitrary system-bath coupling," J. Chem. Phys. 119, 12063-12076 (2003).

${ }^{21}$ M. Cho and R. Silbey, "On the transition from nonadiabatic to adiabatic rate kernel: Schwinger's stationary variational principle and Pade approximation," J. Chem. Phys. 106, 2654-2661 (1997).

${ }^{22}$ M. Sparpaglione and S. Mukamel, "Dielectric friction and the transition from adiabatic to nonadiabatic electron-trasnfer. 1. Solvation dynamics in Liouville space," J. Chem. Phys. 
88, 3263-3280 (1988).

${ }^{23} \mathrm{~S}$. Mukamel, "Collisional broadening of spectral line shapes in two-photon and multiphoton processes," Phys. Rep. 93, 1-60 (1982).

${ }^{24}$ B. Yoon, J. Deutch, and J. Freed, J. Chem. Phys. 62, 4687 (1975).

${ }^{25}$ A. Case David, E. Cheatham Thomas, T. Darden, H. Gohlke, R. Luo, K. M. Merz, A. Onufriev, C. Simmerling, B. Wang, and R. J. Woods, "The amber biomolecular simulation programs," J.Comput. Chem. 26, 1668-1688 (2005).

${ }^{26}$ K. Hinsen, "The molecular modeling toolkit: A new approach to molecular simulations," J. Comput. Chem. 21, 79-85 (2000).

${ }^{27} \mathrm{~J}$. Waser, "Dyadics and the variances and covariances of molecular parameters, including those of best planes," Acta Cryst A 29, 621-631 (1973).

${ }^{28}$ Y. Song and L. L. Dai, "The shear viscosities of common water models by non-equilibrium molecular dynamics simulations," Molecular Simulation 36, 560-567 (2010).

${ }^{29}$ G. Nicholas, B. Anja, L. Anne, P. Francois, B. Martin, and E. Lyndon, "Site-specific backbone dynamics from a crystalline protein by solid-state nmr spectroscopy," J. Am. Chem. Soc. 126, 11422-11423 (2004).

${ }^{30}$ A. Perico and M. Guenza, "Viscoelastic relaxation of segment orientation in dilute polymer solutions," J. Chem. Phys. 83, 3103-3109 (1985).

${ }^{31}$ A. Perico and M. Guenza, "Viscoelastic relaxation of segment orientation in dilute polymer solutions. ii. stiffness dependence of fluorescence depolarization," J. Chem. Phys. 84, 510$516(1986)$.

${ }^{32}$ M. Guenza, "Many chain correlated dynamics in polymer fluids," J. Chem. Phys. 110, 7574-7588 (1999).

${ }^{33}$ K. S. Schweizer, M. Fuchs, G. Szamel, M. Guenza, and H. Tang, "Polymer-mode-coupling theory of the slow dynamics of entangled macromolecular fluids," Macromol. Theory Simul. 6, 1037-1117 (1997).

${ }^{34}$ I. Lyubimov and M. G. Guenza, "First-principle approach to rescale the dynamics of simulated coarse-grained macromolecular liquids," Phys. Rev. E 84, 031801 (2011).

${ }^{35}$ T. K. Leen, R. Friel, and D. Nielsen, "Eigenfunctions of the Multidimensional Linear Noise Fokker-Planck Operator via Ladder Operators," ArXiv (2016), arXiv:1609.01194.

${ }^{36}$ B. Matthias, S. Bouguet-Bonnet, R. Pastor, and A. D. J. MacKerell, "Importance of the cmap correction to the charmm22 protein force field: Dynamics of hen lysozyme," Biophys. 
J. 90, 36-38 (2006).

${ }^{37}$ J. C. Phillips, R. Braun, W. Wang, J. Gumbart, E. Tajkhorshid, E. Villa, C. Chipot, R. D. Skeel, K. Laxmikant, and K. Schulten, "Scalable molecular dynamics with namd," J. Comput. Chem. 26, 1781-1802 (2005), https://onlinelibrary.wiley.com/doi/pdf/10.1002/jcc.20289. 
FIG. 1. Molecular structures of 2-alanine and 8-alanine, obtained via Molecular Mechanics (cfr. I)

FIG. 2. Real and imaginary parts of $j_{00}(\omega)$ for $\mathbf{2}(\mathrm{a})$ and $\mathbf{8}(\mathrm{b})$, obtained via $k=1$ order perturbation method (dotted lines), $k=3$ order perturbation method (dashed lines) and moments method (full lines). The real part of the spectral density is positive, the imaginary part is negative. The red dashed line is shown to easily distinguish positive and negative parts of the ordinate axis.

FIG. 3. Cole-Cole plot of $j_{00}(\omega)$ for 2 (a) and $\mathbf{8}(\mathrm{b}): k=1$ order perturbation method (dotted lines), $k=3$ order perturbation method (dashed lines) and moments method (full lines). $\Re$ and $\Im$ stand, respectively, for the real and imaginary parts of the spectral density.

FIG. 4. Logarithm of the first 50 real eigenvalues, $\alpha_{i}$, of matrix $\mathbf{A}$ for $\mathbf{2}$ (a) and $\mathbf{8}$ (b); the horizontal lines indicate the corresponding values of $d_{i}$ (see Section V, Table III).

FIG. 5. Real part $(\Re)$ of $j_{l l}(\omega)$ with $l, \bar{l}=0, \pm 2$ for $2: k=1$ order perturbation method (dotted lines), $k=3$ order perturbation method (dashed lines) and moments method (full lines).

FIG. 6. Imaginary part $(\Im)$ of $j_{l l}(\omega)$ with $l, \bar{l}=0, \pm 2$ for $2: k=1$ order perturbation method (dotted lines), $k=3$ order perturbation method (dashed lines) and moments method (full lines).

FIG. 7. Real part $(\Re)$ of $j_{l \bar{l}}(\omega)$ with $l, \bar{l}=0, \pm 2$ for $8: k=1$ order perturbation method (dotted lines), $k=3$ order perturbation method (dashed lines) and moments method (full lines).

FIG. 8. Imaginary ( $\Im)$ part of $j_{l l}(\omega)$ with $l, \bar{l}=0, \pm 2$ for $8: k=1$ order perturbation method (dotted lines), $k=3$ order perturbation method (dashed lines) and moments method (full lines).

FIG. 9. Correlation functions of 2-alanine, obtained via molecular dynamics simulations (full lines), and via $k=3$ order perturbation method (dashed lines). The quantities $g_{l, l}(t) / g_{l, l}(0)$ are reported.

FIG. 10. Correlation functions of 8-alanine, obtained via molecular dynamics simulations (full lines), and via $k=3$ order perturbation method (dashed lines). The quantities $g_{l, l}(t) / g_{l, l}(0)$ are reported.

FIG. 11. Visual representation of potential coupling of two selected torsional angles (a and b) of 8: the selected bonds are indicated in cyan, while the coupling with other torsional coordinates is represented via a color code (red: high value of $\left|\omega_{K}\right|_{i j}$, green: low value of $\left|\omega_{K}\right|_{i j}$.

FIG. 12. Visual representation of friction coupling of two selected torsional angles (a and b) of 8: the selected bonds are indicated in cyan, while the coupling with other torsional coordinates is represented via a color code (red: high value of $\left|\omega_{K}\right|_{i j}$, green: low value of $\left|\omega_{K}\right|_{i j}$. 

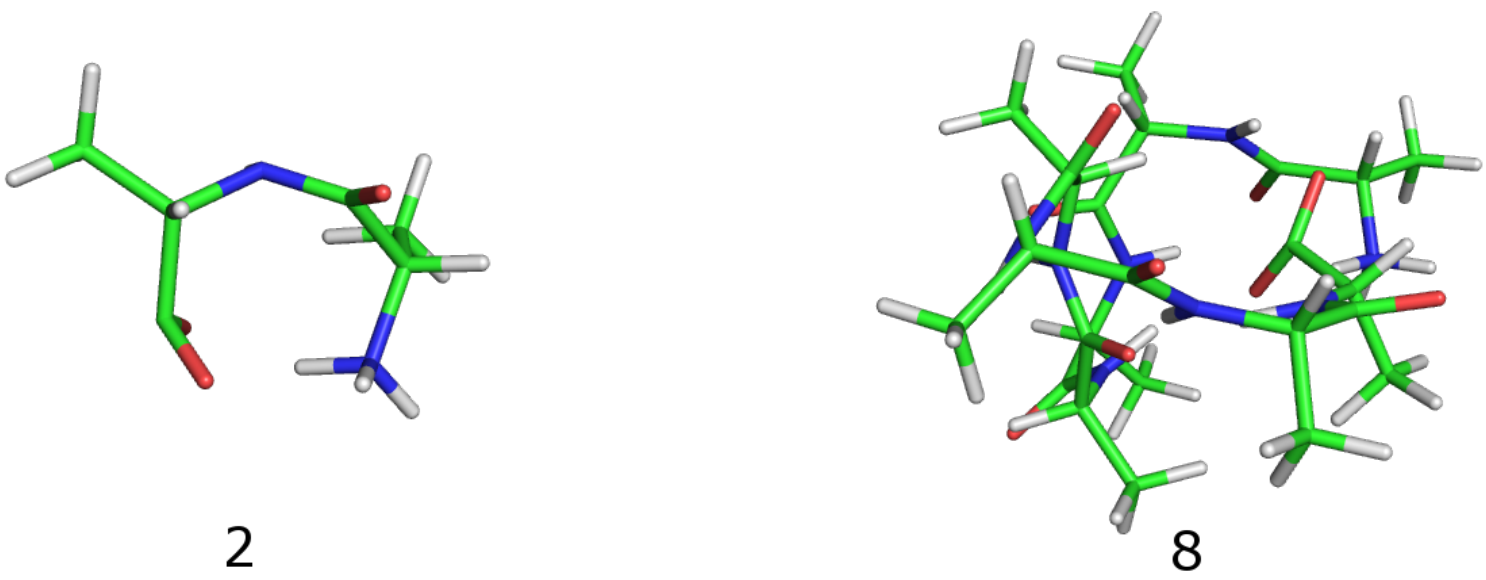

Figure 1 

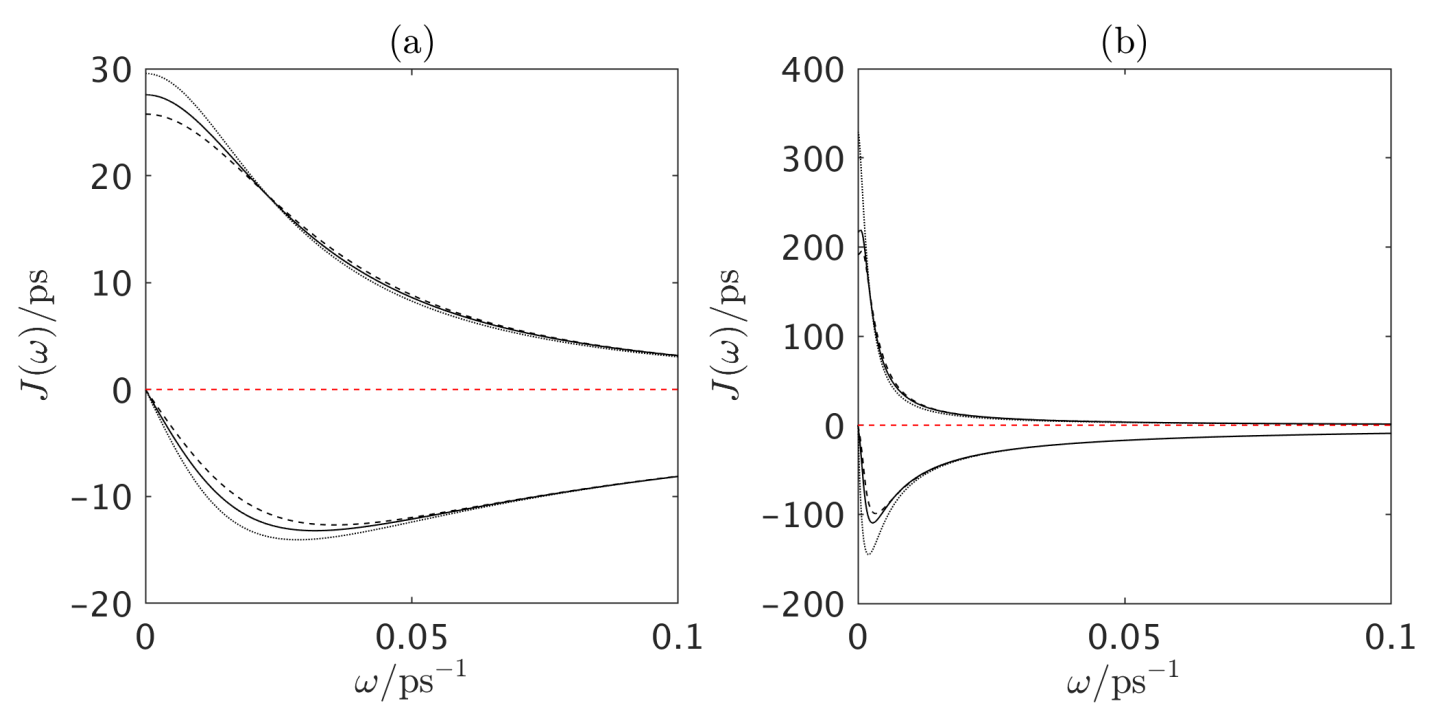

Figure 2 
(a)

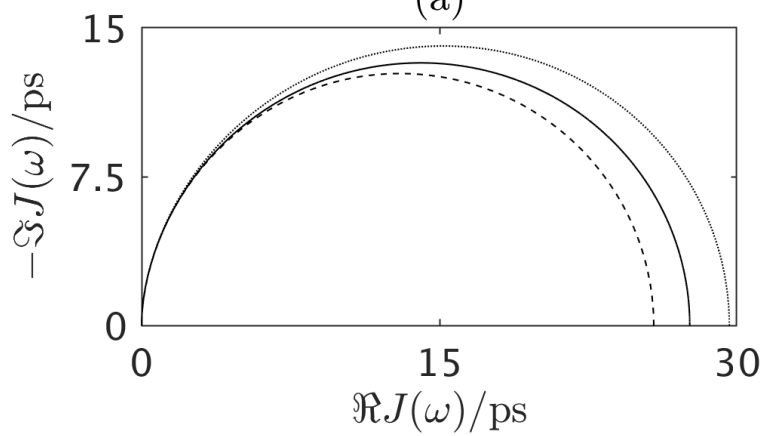

(b)

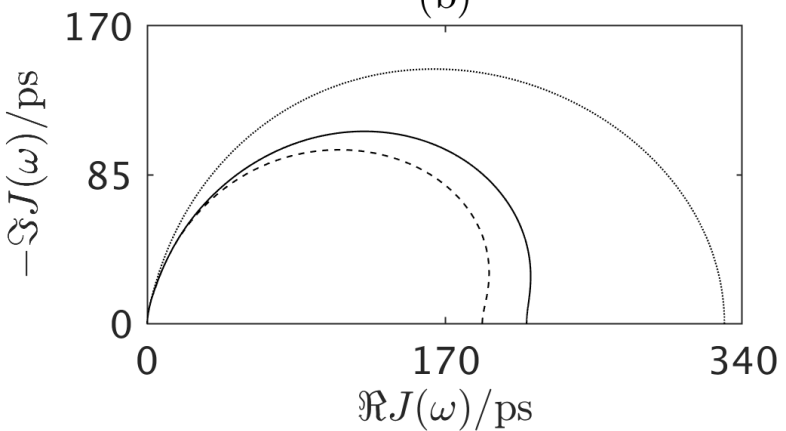

Figure 3 
(a)

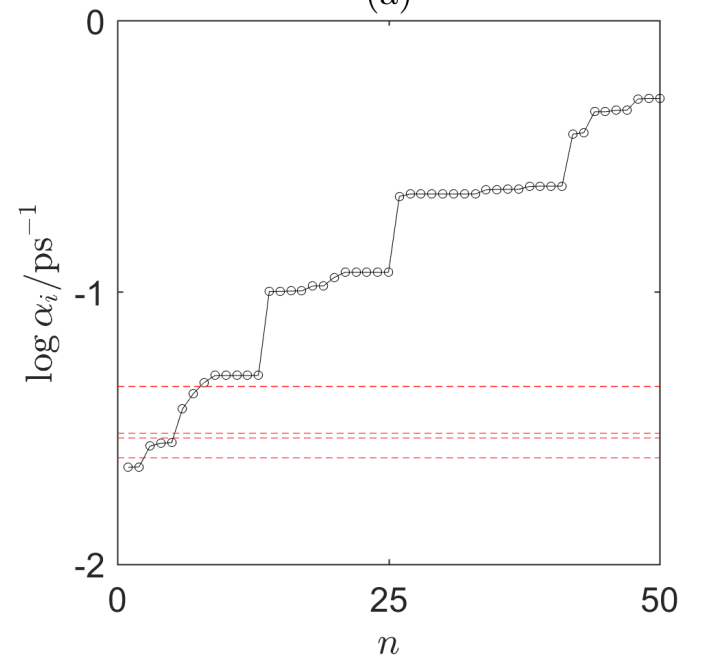

(b)

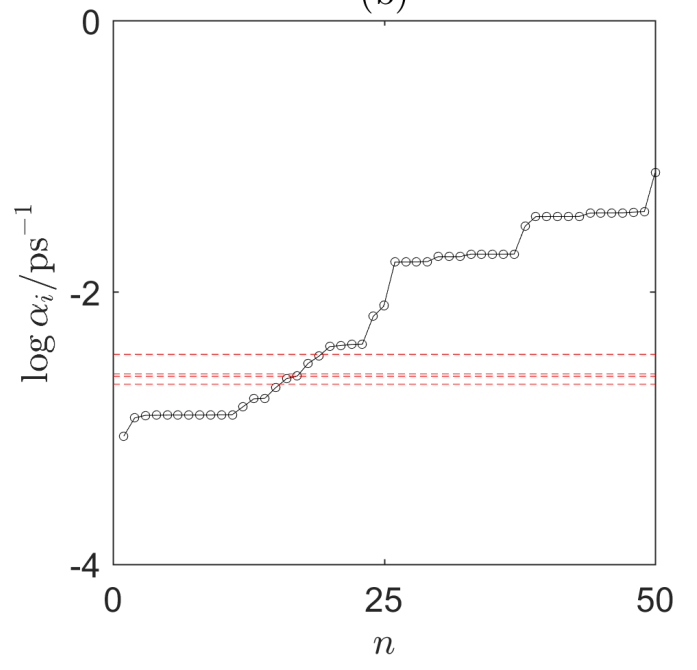

Figure 4 

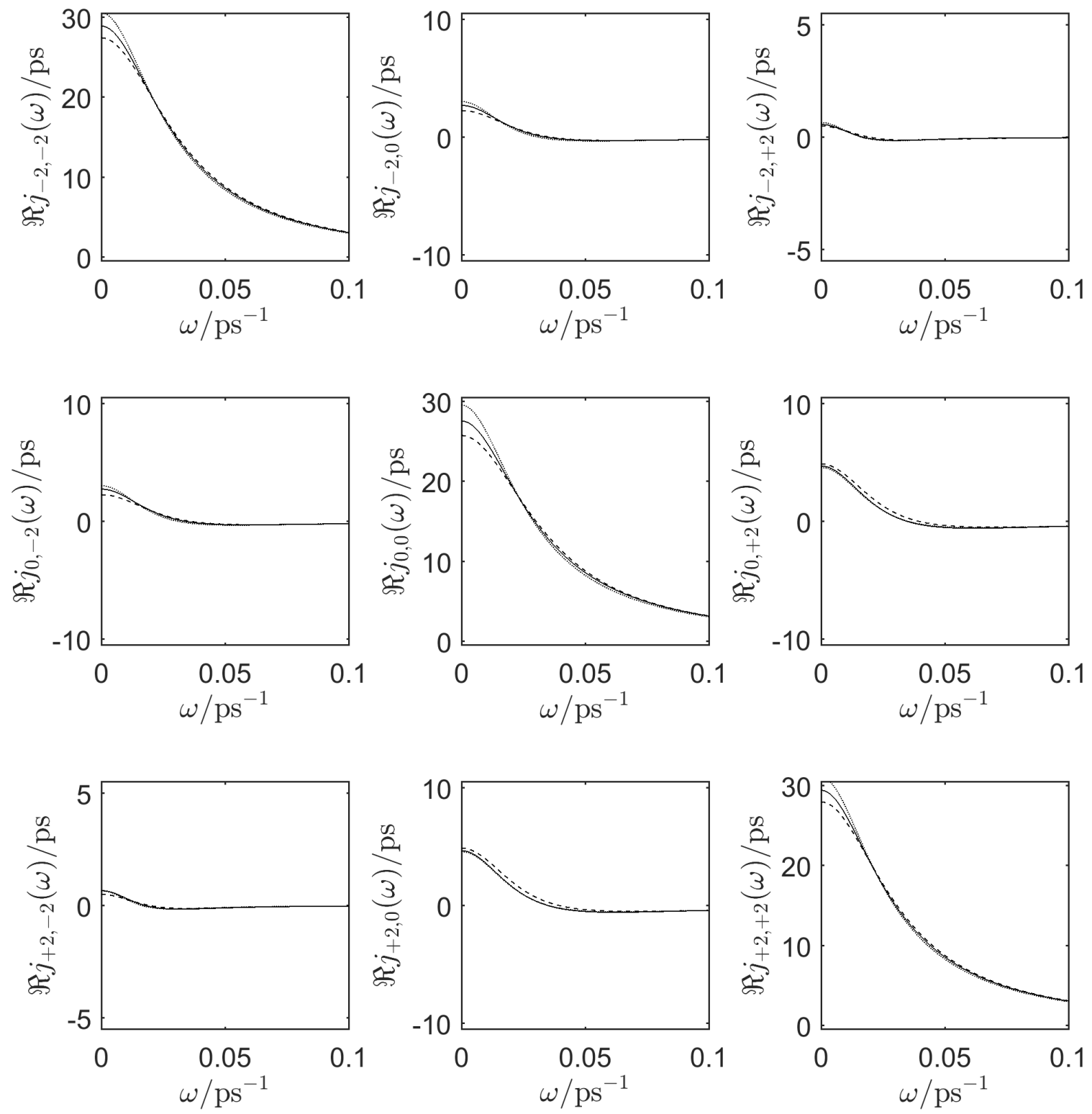

Figure 5 

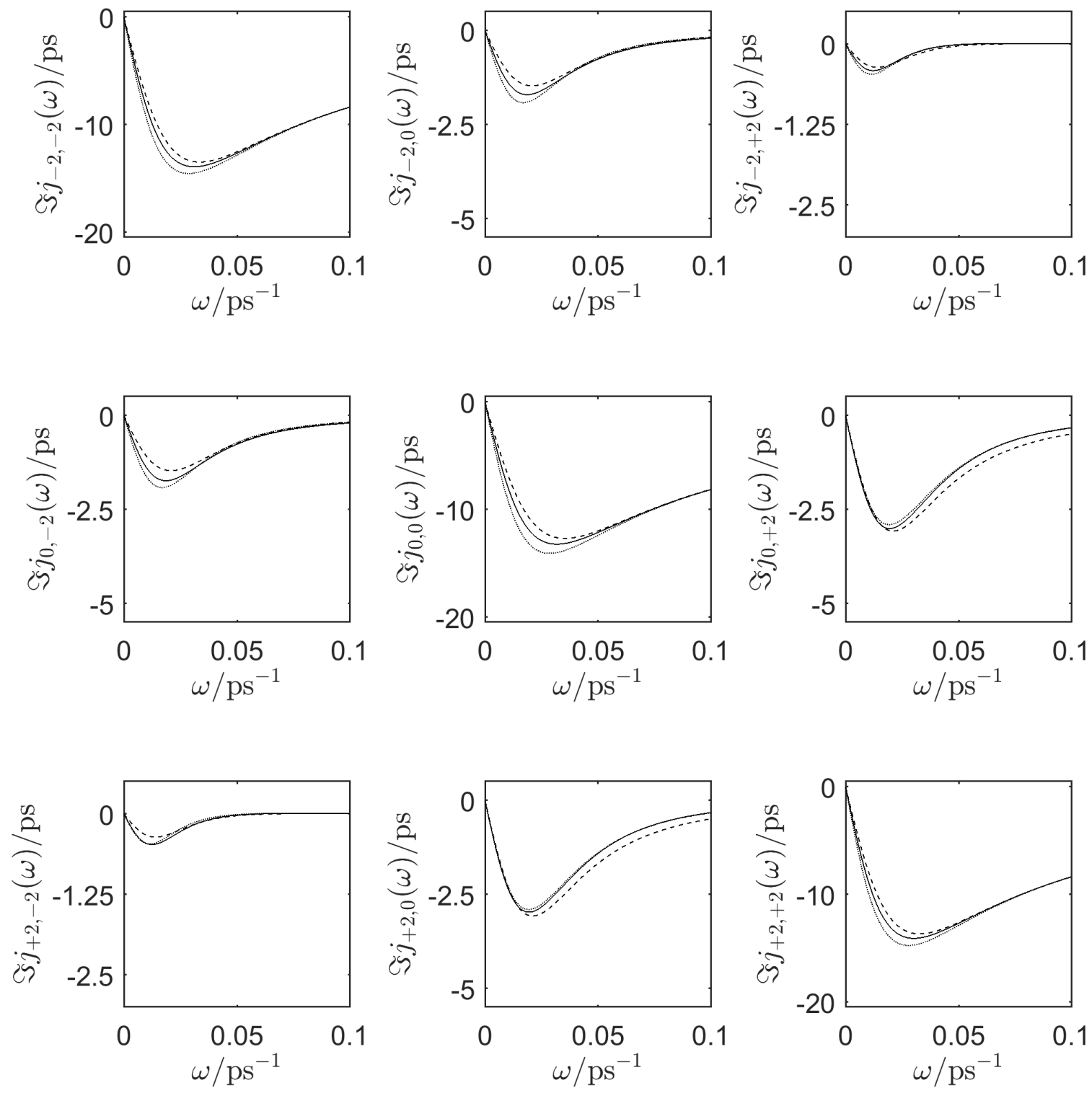

Figure 6 

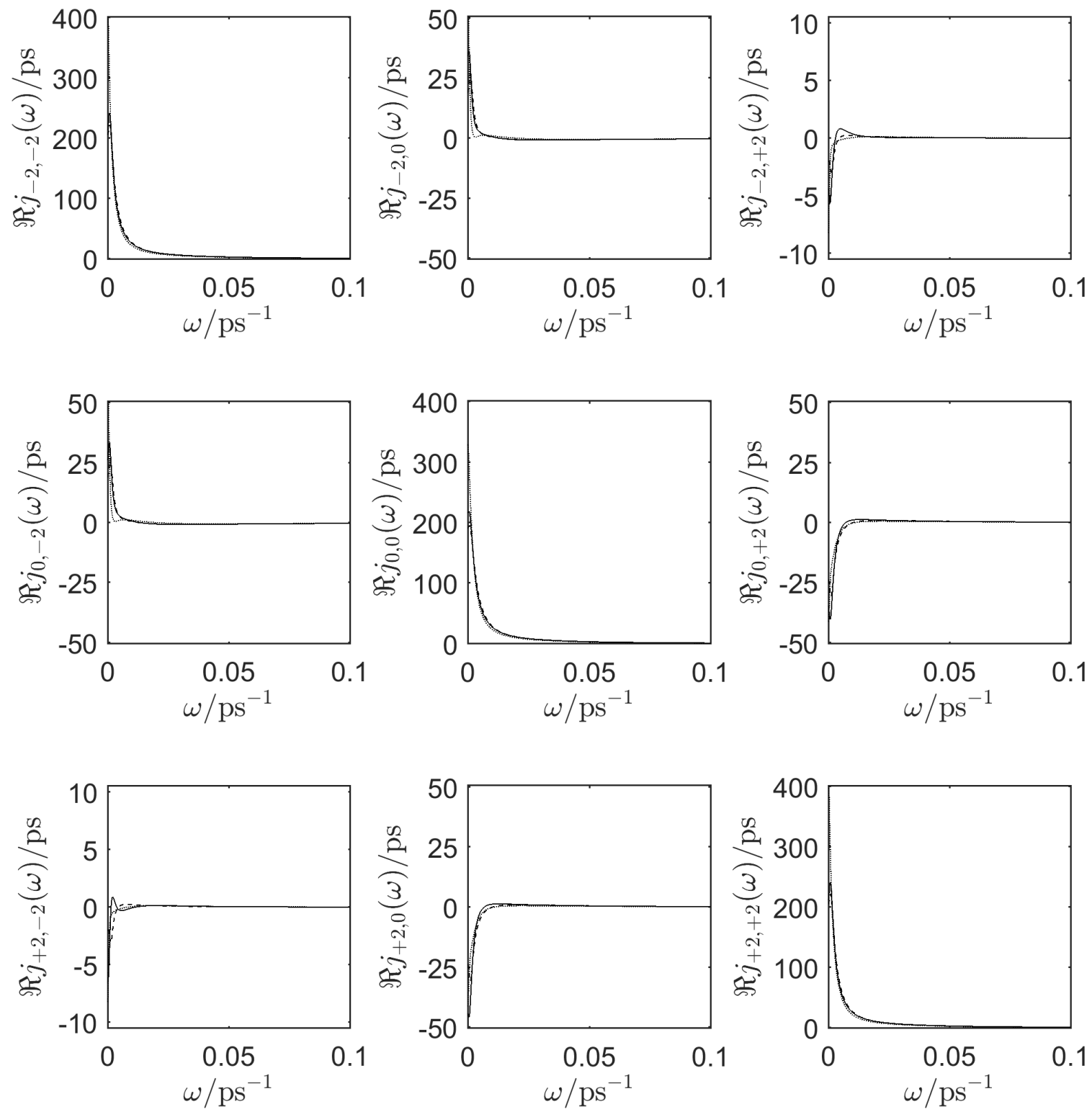

Figure 7 

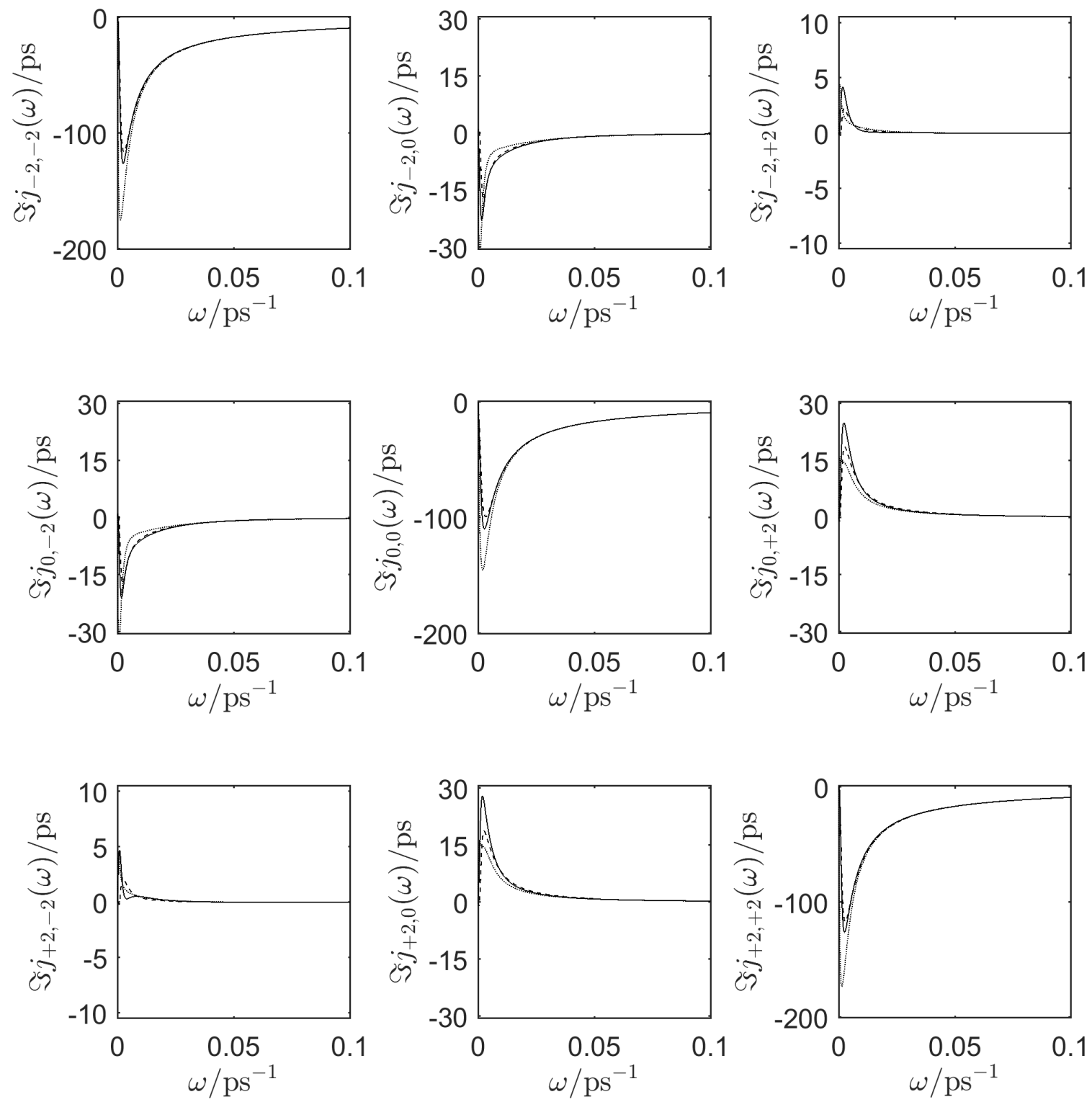

Figure 8 

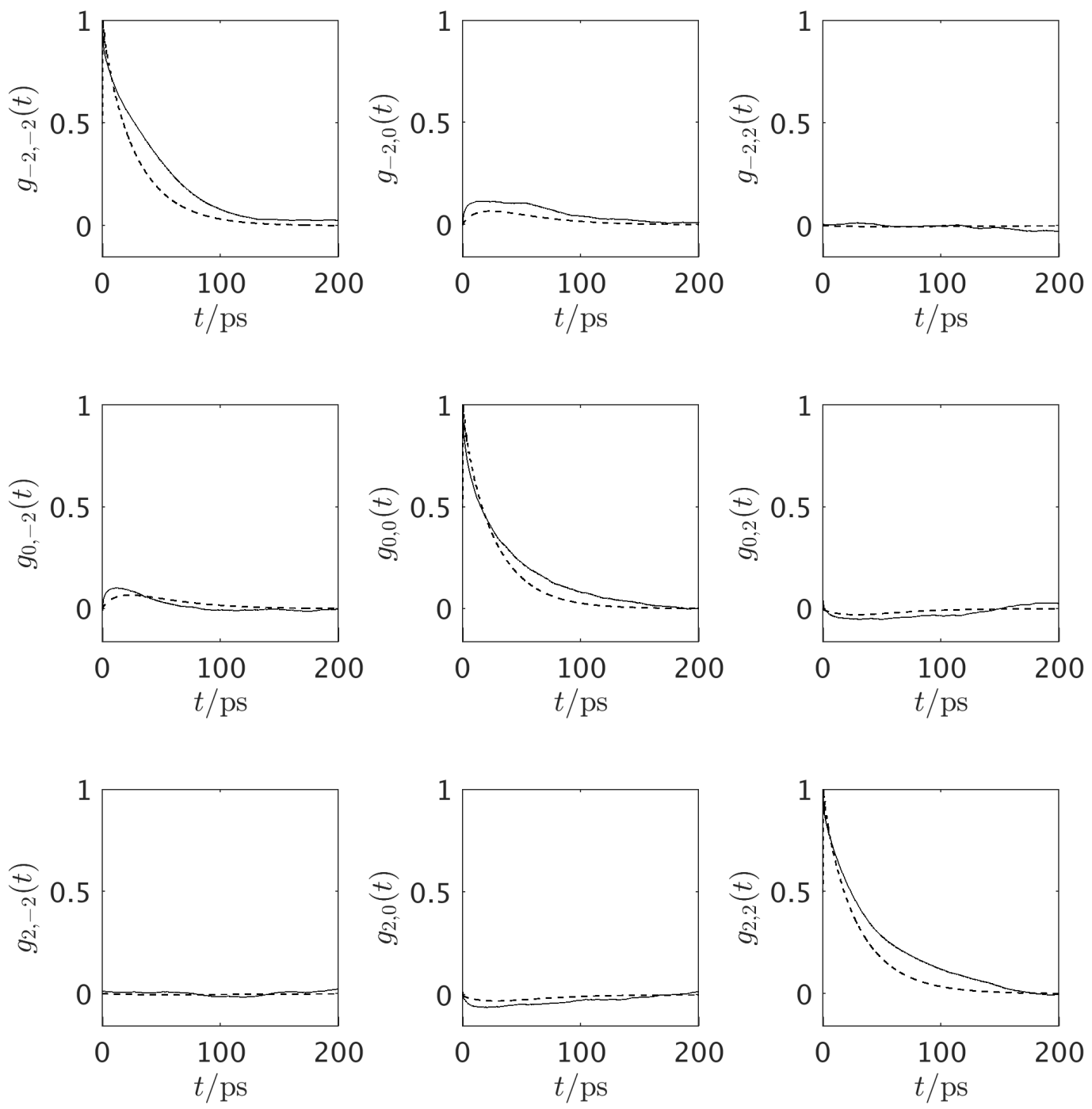

Figure 9 

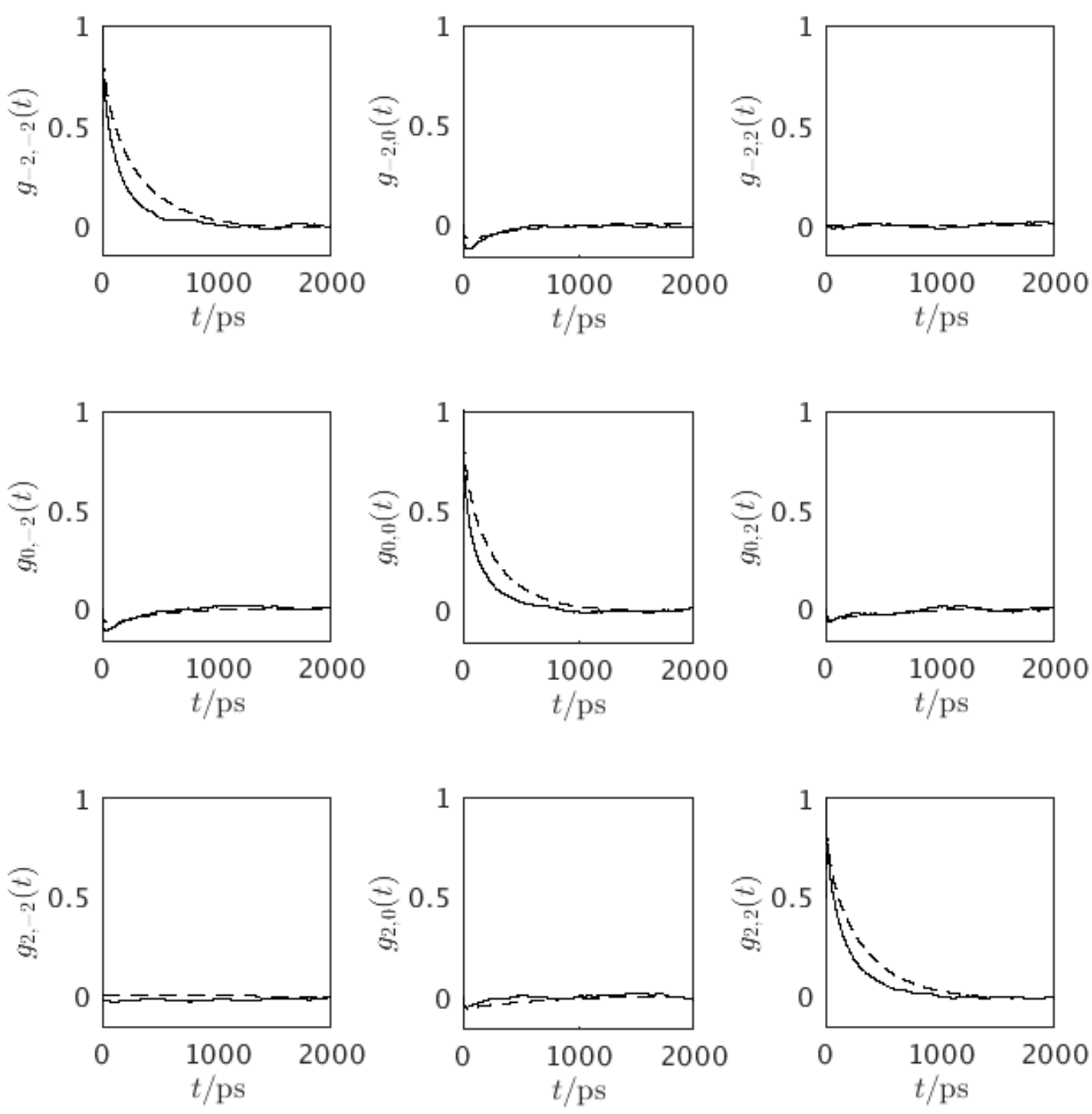

Figure 10 

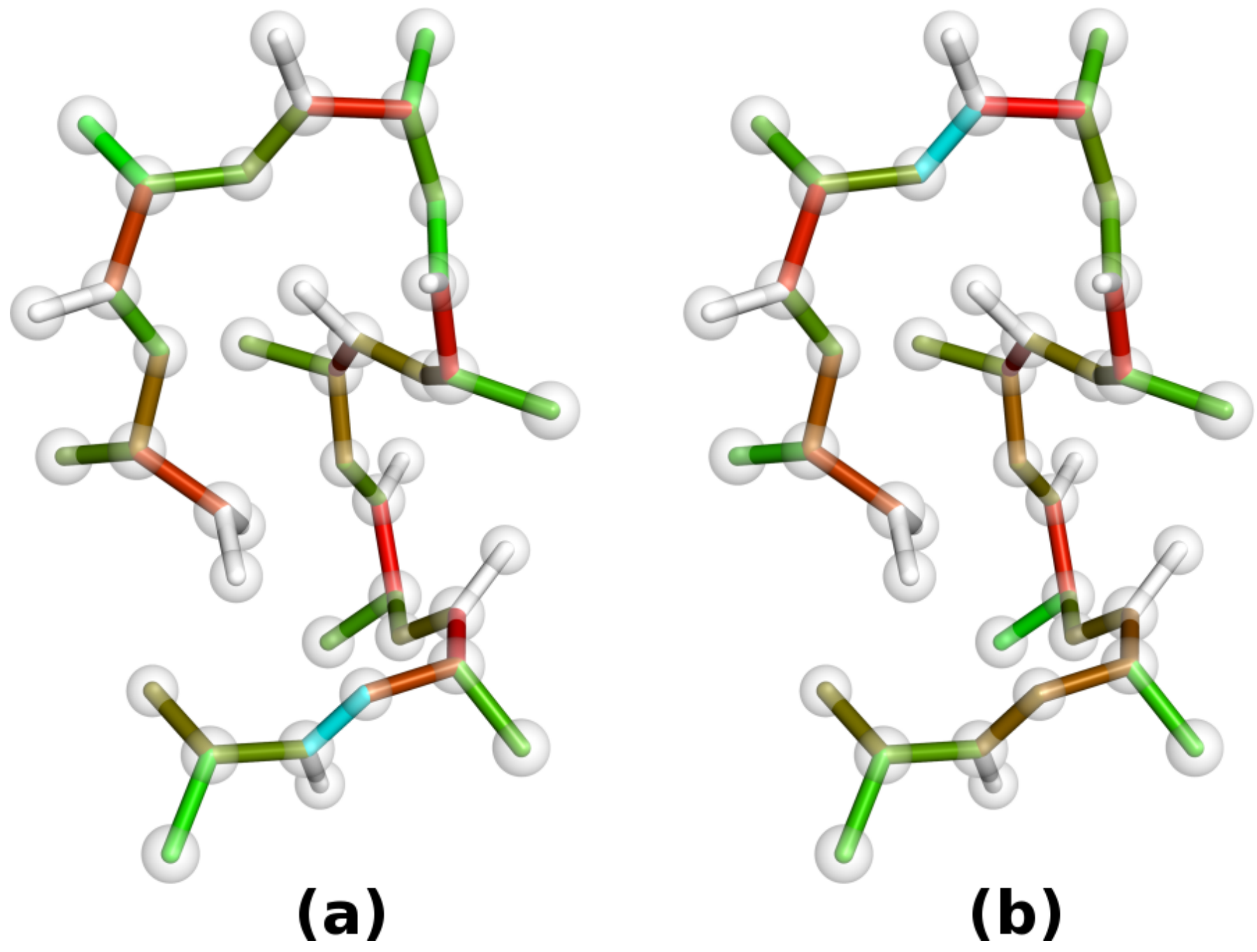

Figure 11 

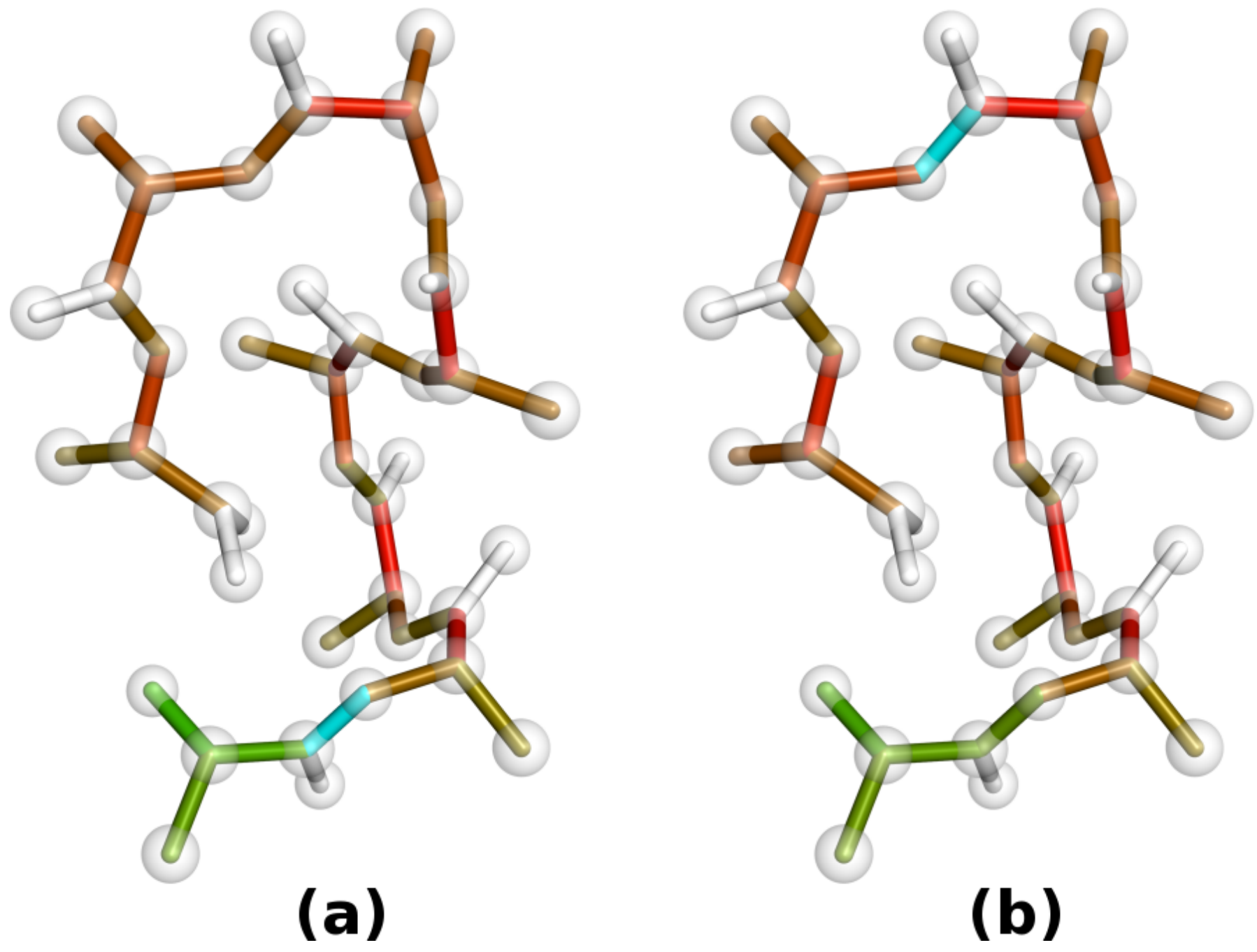

Figure 12 